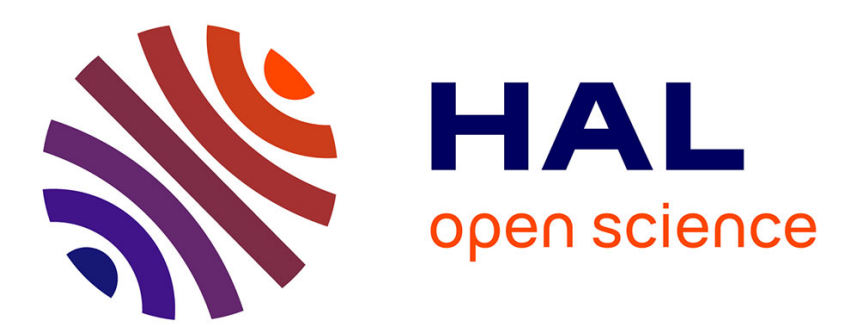

\title{
Effective models for reactive flow under a dominant Peclet number and order one Damköhler number: Numerical simulations
}

\author{
Catherine Choquet, Carole Rosier
}

\section{- To cite this version:}

Catherine Choquet, Carole Rosier. Effective models for reactive flow under a dominant Peclet number and order one Damköhler number: Numerical simulations. Nonlinear Analysis: Real World Applications, 2012, pp.10.1016/j.nonrwa.2012.02.008. 10.1016/j.nonrwa.2012.02.008 . hal-00823357

\section{HAL Id: hal-00823357 \\ https://hal.science/hal-00823357}

Submitted on 20 May 2013

HAL is a multi-disciplinary open access archive for the deposit and dissemination of scientific research documents, whether they are published or not. The documents may come from teaching and research institutions in France or abroad, or from public or private research centers.
L'archive ouverte pluridisciplinaire HAL, est destinée au dépôt et à la diffusion de documents scientifiques de niveau recherche, publiés ou non, émanant des établissements d'enseignement et de recherche français ou étrangers, des laboratoires publics ou privés. 


\title{
Effective models for reactive flow under dominant Péclet number and order one Damköhler number: numerical simulations
}

\author{
Catherine Choquet $^{\mathrm{a}}$, Carole Rosier ${ }^{\mathrm{b}}$ \\ ${ }^{a}$ Corresponding author: Université de La Rochelle, MIA CNRS EA 3165, Avenue Michel \\ Crépeau, F-17042 La Rochelle, France, cchoquet@univ-lr.fr \\ ${ }^{b}$ Univ. Lille Nord de France, F-59000, Lille, France, ULCO, LMPA J. Liouville, BP \\ 699, F-62 228 Calais, France, CNRS FR 2956, carole.rosier@lmpa.univ-littoral.fr
}

\begin{abstract}
The paper is devoted to the longitudinal dispersion of a soluble substance released in a steady laminar flow through slit channel with heterogeneous reaction at the outer wall. The reactive transport happens in presence of dominant Péclet number and order one Damköhler number. In particular, these Péclet numbers correspond to Taylor's dispersion regime. An effective model for the enhanced diffusion in this context was derived recently . It contains memory effects and contributions to the effective diffusion and effective advection velocity, due to the flow and chemistry reaction regime. In the present paper, we show through numerical simulations the efficiency of this new model. In particular, using Taylor's 'historical' parameters, we illustrate that our derived contributions are important and that using them is necessary in order to simulate correctly the reactive flows. We emphasize three main points. First, we show how the effective diffusion is enhanced by chemical effects at dispersive times. Second, our model captures an intermediate regime where the diffusion is anomalous and the distribution is asymmetric. Third, we show how the chemical effects also slow down the average speed of the front.
\end{abstract}

Keywords: Taylor's dispersion, memory effects, enhanced diffusion, anomalous diffusion, finite elements.

35B25, 92E20, 76F25, 65N30, 76M10.

\section{Introduction}

We consider the evolution of a soluble substance introduced into the Poiseuille flow in a slit channel. In fact, this problem could be studied in 
three distinct regimes: a) diffusion-dominated mixing; b) Taylor dispersionmediated mixing; c) chaotic advection. We focus our analysis to regime b), corresponding to dominant Péclet's numbers, but smaller than a threshold value. We also consider the transition between $a$ ) and $b$ ).

If the channel is the domain $\Omega^{*}$,

$$
\Omega^{*}=\left\{\left(x^{*}, y^{*}\right): x^{*} \in \mathbb{R},\left|y^{*}\right|<H\right\},
$$

the height of the channel being denoted by $H, H>0$, the equation governing the evolution of the solute concentration $c^{*}$ is:

$$
\frac{\partial c^{*}}{\partial t^{*}}+q\left(y^{*}\right) \frac{\partial c^{*}}{\partial x^{*}}-D^{*} \frac{\partial^{2} c^{*}}{\partial\left(x^{*}\right)^{2}}-D^{*} \frac{\partial^{2} c^{*}}{\partial\left(y^{*}\right)^{2}}=0 \quad \text { in } \Omega^{*}
$$

where $q(z)=Q^{*}\left(1-(z / H)^{2}\right)$ is the Poiseuille profile, $Q^{*}$ being the maximum velocity at the axis, while $D^{*}$ is the molecular diffusion. Equation (1) is of course completed by appropriate initial and boundary conditions. Boundary conditions are especially important in the present work since they model the reactions with the walls. We will turn back to this point somewhat later. Let us first focus on equation (1) and introduce appropriate scales to emphasize the significance of a dominant Péclet number. The obvious transversal length scale is $H$. For all other quantities we use reference values denoted by the subscript $R$. Setting

$$
\left\{\begin{array}{l}
c=\frac{c^{*}}{c_{R}}, x=\frac{x^{*}}{L_{R}}, y=\frac{y^{*}}{H}, t=\frac{t^{*}}{T_{R}}, \\
Q=\frac{Q^{*}}{Q_{R}}, D=\frac{D^{*}}{D_{R}}
\end{array}\right.
$$

where $L_{R}$ is the 'observation distance', we obtain the dimensionless equation

$$
\frac{\partial c}{\partial t}+\frac{Q_{R} T_{R}}{L_{R}} Q\left(1-y^{2}\right) \frac{\partial c}{\partial x}-\frac{D_{R} T_{R}}{L_{R}^{2}} D \frac{\partial^{2} c}{\partial x^{2}}-\frac{D_{R} T_{R}}{H^{2}} D \frac{\partial^{2} c}{\partial y^{2}}=0
$$

in

$$
\Omega=\mathbb{R} \times(-1,1) .
$$

The times scales involved in this equation are

$$
\begin{aligned}
& T_{L}=\text { characteristic longitudinal time scale }=\frac{L_{R}}{Q_{R}}, \\
& T_{T}=\text { characteristic transversal time scale }=\frac{H^{2}}{D_{R}} .
\end{aligned}
$$


We define a small parameter $\varepsilon$ by

$$
\varepsilon=\frac{H}{L_{R}} .
$$

The Péclet number Pe, defined by

$$
\mathbf{P e}=\frac{L_{R} Q_{R}}{D_{R}},
$$

being supposed to be dominant but before a threshold, assume there exists $0<\alpha<2$ such that $\mathbf{P e}=\varepsilon^{-\alpha}$. The lower order process of diffusion is rescaled by $\frac{T_{T}}{T_{L}}=\frac{H Q_{R}}{D_{R}} \varepsilon=\mathcal{O}\left(\varepsilon^{2-\alpha}\right)$. Choosing the dispersive time for reference time by setting

$$
T_{R}=T_{L},
$$

equation (2) yields:

$$
\frac{\partial c^{\varepsilon}}{\partial t}+Q\left(1-y^{2}\right) \frac{\partial c^{\varepsilon}}{\partial x}=D \varepsilon^{\alpha} \frac{\partial^{2} c^{\varepsilon}}{\partial x^{2}}+D \varepsilon^{\alpha-2} \frac{\partial^{2} c^{\varepsilon}}{\partial y^{2}} \quad \text { in } \Omega .
$$

Clearly, there is a great anisotropy in the former equation. The dispersion is due to this combined action of dominant convection parallel to the axis and molecular diffusion in the radial direction. Actually, in the fundamental papers [40] and [1], Taylor and Aris found the following one-dimensional approximation for (1) with no-flux (Neumann) boundary conditions on the side walls:

$$
\partial_{t^{*}} c^{T a y}+\frac{2 Q^{*}}{3} \partial_{x^{*}} c^{T a y}-D^{T a y} \partial_{x^{*}}^{2} c^{T a y}=0 \text { in } \mathbb{R}_{+} .
$$

In (4), the effective axial diffusivity contains a contribution proportional to the square of the transversal Péclet number in addition to the original molecular diffusivity:

$$
D^{T a y}=D^{*}\left(1+\frac{8}{945} \mathbf{P e}_{T}^{2}\right), \quad \mathbf{P e}_{T}=\frac{Q^{*} H}{D^{*}} .
$$

The solute is convected by the average velocity of the flow $\langle q\rangle=2 Q^{*} / 3$ and diffuses with respect to the Taylor's dispersion coefficient $D^{\text {Tay }}$.

Taylor's paper [40] was the first one of a huge literature on the subject. This does not allows us to give an exhaustive list of references. Let us only mention the mostly used methods. Roughly speeking, passing from the 2D-equation (1) to the 1D-equation (4) corresponds to a vertical averaging. 
Difficulty is that the equation for the difference between physical and averaged concentrations is not closed because of the dispersive source term $\partial_{x}\left\langle q c^{*}\right\rangle$. For its determination one should go to the next order and actually solve an infinite system of equations. Derivation of (4) is thus linked with the choice of 'closure scheme'. Aris [1] presented a formal derivation of Taylor's approximation using the method of moments. This method, further developed by Brenner and collaborators (e.g. [7]) is still very common nowadays. Other interesting series expansion methods are in the works [20] [22] and [39]. Important step is to "justify" the closure of the computations (or the truncation of the series expansion) either by orders of magnitude arguments (e.g. [19], [41], [6]), or by homogenization techniques (e.g. [36]) or volume averaging approach (e.g. [35]; this latter approach being adapted for complex geometries) or by a center manifold argument (e.g. [27]). Very different approaches consist in starting from the position process of each molecule with an appropriate description of its transition probability function (e.g. [4]). Taylor-Aris result (4) is then equivalent to the asymptotic Gaussian nature of the process. Such a probabilistic analysis may lead to a variety of non-local transport models (e.g. [28]). In particular, some abstract assumptions on the transitions between jumps of the molecules lead to kernel memory terms comparable to those used in the present paper, though derived with a very different method. We finally mention derivation of (reversible) non-parabolic models, using statistical physics, in the papers $[9,10,11]$ and $[5]$.

The approximate models proposed in the latter references are justified for asymptotic large times. Nethertheless it is worth mentioning that the literature does not provide information about the induced error. Up to our knowledge, the articles $[29,30,14,15]$ give the first derivations of Taylor's type models controlling the error in energy, without assuming unrealistically regular data. More precisely, in the latter papers, the optimization of the error estimates is a crucial step in predicting the effective coefficients.

Moreover papers $[29,30,14,15]$ focus on possible chemical effects. One may guess of course that adsorption and desorption processes increase the heterogeneity of the solute plume injected in the pipe and thus should influence the final dispersion phenomenon. Flow with chemistry has already been considered in numerous works, based on the same methods than the ones listed above, and thus without error estimates. We mention for instance $[3,16],[23]$ (for a reactive mixture, but no reactions with the walls) based on the method of moments, [32] based on the Fife and Nicholes expansion, [38] based on [37], [33] based on closure schemes of turbulence modeling and a volume averaging approach, [2] based on the center manifold approach. 
The present paper aims at giving numerical illustrations of the results obtained in [15] where adsorption and desorption reactions occur on the

walls of the channel through a linear driving force model with a finite kinetics and a linear isotherm. In [15], the authors derive rigorously an upscaled Taylor's type model where influence of the chemical kinetics on the dispersive effects is explicit. The model was justified through error estimates depending explicitly on the small parameter $\varepsilon=H / L_{R}$. Presence of an initial time boundary layer allows only a global error estimate in $L^{2}$ with respect to space and time.

In the present paper, we show through numerical simulations the efficiency of the model derived in [15]. In particular, using Taylor's original parameters (see Section $\S 3$ ), we illustrate that the derived contributions are important and that using them is necessary in order to simulate correctly the reactive flows (see Subsection 4.1). The model derived in [15] is reproduced in Section $\S 2$ (see (9)-(12)). The reader will note that the chemical reactions on the walls produce complicated memory terms. We thus aim to emphasize three main points through our numerical simulations. First, in Subsection 4.2, we turn to the question of anomalous diffusion. We show how the effective diffusion is enhanced by chemical effects at dispersive times. Furthermore the model of [15] also captures an intermediate regime where the diffusion is anomalous and the distribution is asymmetric. Second, in Subsection 4.3, we show how the chemical effects also slow down the average speed of the front. Finally, in Section $\S 5$, we present numerical illustrations of some other effective models: the case with Danckwerts boundary condition in subsection 5.1, and the case of nonlinear reactions in subsection 5.2 .

\section{Exact and upscaled problems}

Let us write the precise setting of the problem. We consider the transport of a reactive solute by diffusion and convection by Poiseuille's velocity in an infinite 2D channel. The solute particles do not react among themselves. Instead they undergo an adsorption process at the lateral boundary. For a general discussion on the modeling of adsorption processes in porous media we refer to [17] and [24]. 
We consider the following exact model for the solute concentration $c^{*}$ :

$$
\begin{aligned}
& \frac{\partial c^{*}}{\partial t^{*}}+q\left(y^{*}\right) \frac{\partial c^{*}}{\partial x^{*}}-D^{*} \frac{\partial^{2} c^{*}}{\partial\left(x^{*}\right)^{2}}-D^{*} \frac{\partial^{2} c^{*}}{\partial\left(y^{*}\right)^{2}}=0 \quad \text { in } \Omega^{*}, \\
& -D^{*} \partial_{y^{*}} c^{*}=\frac{\partial c_{s}^{*}}{\partial t^{*}}=k_{s}^{*}\left(c^{*}-\frac{c_{s}^{*}}{K_{e}^{*}}\right) \quad \text { on } \Gamma^{*}, \\
& c^{*}\left(x^{*}, y^{*}, 0\right)=c_{00}^{*}\left(x^{*}\right), \quad c_{s}^{*}\left(x^{*}, 0\right)=c_{s 0}^{*}\left(x^{*}\right) .
\end{aligned}
$$

The crucial difference between problem (6)-(8) and the the one originally considered by Taylor is the boundary condition on the side walls $\Gamma^{*}=$ $\left\{\left(x^{*}, y^{*}\right): x^{*} \in \mathbb{R},\left|y^{*}\right|=H\right\}$. Taylor assumed no-flux conditions. Here equation (7) describes reaction at channel wall $\Gamma^{*}$ linking the solute concentration $c^{*}$ and the adsorbed species surface concentration $c_{s}^{*}$. The adsorption rate constant is $k_{s}^{*}$ (homogeneous to a velocity), the linear adsorption equilibrium constant is $K_{e}^{*}$ (length), the constant desorption rate being characterized by $k_{s}^{*} / K_{e}^{*}$. These quantities are all positive real numbers. Equation (8) describes initial infiltration with a mollified Dirac pulse of water containing a solute of volume concentration $c_{00}^{*}$ and the adsorbed species surface concentration $c_{s 0}^{*}$. In [15], we assume $c_{00}^{*} \in C_{0}^{\infty}(\mathbb{R}), c_{00}^{*} \geq 0$ and $c_{s 0}^{*} \in C_{0}^{\infty}(\mathbb{R})$, $c_{s 0}^{*} \geq 0$. Following Taylor's example (B1) (see [40], page 192), we can take for $c_{00}^{*}$ the mollified Dirac measure of mass M, concentrated at $x=0$.

We have already explained in the Introduction how appropriate scales have to be chosen ensuring the Péclet number in the range of the Taylor's dispersion regime (see the derivation of (3)). We now have to describe the order of magnitude of the kinetics, characterized by the Damköhler number defined below. If we introduce reference values denoted by the subscript $R$ in the reaction equation (7), three characteristic time scales appear,

$$
\begin{aligned}
& T_{D e}=\text { characteristic desorption time scale }=\frac{K_{e R}}{k_{s R}}, \\
& T_{A}=\text { characteristic adsorption time scale }=\frac{c_{s R}}{c_{R} k_{s R}}, \\
& T_{\text {react }}=\text { superficial chemical reaction time scale }=\frac{H}{k_{s R}},
\end{aligned}
$$

with the Damköhler number defined by

$$
\mathbf{D a}=\frac{L_{R}}{T_{A} Q_{R}} .
$$

Assuming $K_{e R} \approx H$, and the time scales $T_{D e}, T_{A}$ and $T_{L}$ of the same order, implies Damköhler's number of order one (with respect to $\varepsilon$, that is of order $\varepsilon^{0}$ ). We will see below that this order one (to be compared with the order 
$\varepsilon^{\alpha}, \alpha>0$, of the Péclet number) is sufficient to ensure that the kinetics appear at the main scale in the upscaled model. Hence one should take care simultaneously of the flow and of the chemical reactions.

It should be noted that transport models derived for nonreactive solutes are often used, in conjunction with experimentally-derived correctors, to predict the transport of reactive chemicals. Here instead we used an upscaled model rigorously derived in [15]. In [15], the authors prove that the upscaled problem in $\mathbb{R}_{+}$corresponding to (6)-(8) for the basic dimensional effective concentration $c^{e f f}$ is the following: find $c^{e f f} \in L^{2}((0, T) \times \mathbb{R})$ such that

$$
\begin{aligned}
& \frac{\partial c^{e f f}}{\partial t^{*}}+\frac{2}{3} Q^{*} \frac{\partial c^{e f f}}{\partial x^{*}}-D^{*}\left(1+\frac{8}{945} \mathbf{P e}_{T}^{2}\right) \frac{\partial^{2} c^{e f f}}{\partial\left(x^{*}\right)^{2}} \\
& +\frac{k_{s}^{*}}{K_{e}^{*}} c^{e f f}-\left(\frac{k_{s}^{*}}{K_{e}^{*}}\right)^{2} \int_{0}^{t^{*}} e^{-k_{s}^{*}\left(t^{*}-\xi\right) / K_{e}^{*}} c^{e f f}(\cdot, \xi) d \xi \\
& -\frac{1}{3} \mathbf{P e}_{T} \mathbf{D} \mathbf{a}_{T} \frac{H}{K_{e}^{*}} \frac{k_{s}^{*}}{K_{e}^{*}}\left(c^{e f f}\right. \\
& \left.+\int_{0}^{t^{*}} e^{-k_{s}^{*}\left(t^{*}-\xi\right) / K_{e}^{*}}\left(\left(\frac{k_{s}^{*}}{K_{e}^{*}}\right)^{2}\left(t^{*}-\xi\right)-2 \frac{k_{s}^{*}}{K_{e}^{*}}\right) c^{e f f}(\cdot, \xi) d \xi\right) \\
& +\frac{4}{45} \frac{H}{K_{e}^{*}} \mathbf{P e}_{T} k_{s}^{*}\left\{\partial_{x} c^{e f f}-\frac{k_{s}^{*}}{K_{e}^{*}} \int_{0}^{t^{*}} e^{-k_{s}^{*}\left(t^{*}-\xi\right) / K_{e}^{*}} \partial_{x} c^{e f f}(\cdot, \xi) d \xi\right\} \\
& =\frac{k_{s}^{*}}{K_{e}^{*}} e^{-k_{s}^{*} t^{*} / K_{e}^{*}}\left\{\frac{c_{s 0}^{*}}{K_{e}^{*}}+\frac{1}{3} \mathbf{P e}_{T} \mathbf{D a}_{T} \frac{c_{s 0}^{*}}{K_{e}^{*}} \frac{H}{K_{e}^{*}}\left(\frac{k_{s}^{*}}{K_{e}^{*}} t^{*}-1\right)\right\} \\
& +e^{-k_{s}^{*} t^{*} / K_{e}^{*}} \frac{4}{45} \frac{k_{s}^{*}}{K_{e}^{*}} \frac{H}{K_{e}^{*}} \mathbf{P e}_{T} \partial_{x} c_{s 0}^{*}, \\
& c_{\mid t=0}^{e f f}=c_{00}^{*},
\end{aligned}
$$

where the transversal Péclet et Damköhler numbers, $\mathbf{P e}_{T}$ and $\mathbf{D a} \mathbf{a}_{T}$ are given by

$$
\mathbf{P e}_{T}=\frac{Q^{*} H}{D^{*}} \quad \text { and } \quad \mathbf{D a}_{T}=\frac{k_{s}^{*}}{Q^{*}} .
$$

Existence of a unique solution $c^{e f f} \in H^{1}\left(0, T ; H^{2}(\mathbb{R})\right.$ is proved in [15]. Then 
the dimensional effective solute and sorbed concentrations read

$$
\begin{gathered}
c_{f u l l}^{\text {eff }}\left(x^{*}, y^{*}, t^{*}\right)=c^{e f f}\left(x^{*}, t^{*}\right) \\
\left.+H \mathbf{P e}_{T} \partial_{x} c^{e f f}\left(x^{*}, t^{*}\right)\left(\frac{1}{6} \frac{y^{* 2}}{H^{2}}-\frac{1}{12} \frac{y^{* 4}}{H^{4}}\right)-\frac{7}{180}\right) \\
+\left(\frac{1}{6}-\frac{y^{* 2}}{2 H^{2}}\right) \mathbf{P e}_{T} \mathbf{D} \mathbf{a}_{T} \frac{H}{K_{e}^{*}}\left(c^{e f f}\left(x^{*}, t^{*}\right)-\frac{k_{s}^{*}}{K_{e}^{*}} \int_{0}^{t^{*}} e^{-k_{s}^{*}\left(t^{*}-\xi\right) / K_{e}^{*}} c^{e f f}\left(x^{*}, \xi\right) d \xi\right) \\
-\left(\frac{1}{6}-\frac{1}{2} \frac{y^{* 2}}{H^{2}}\right) \frac{c_{s 0}^{*}}{K_{e}^{*}} e^{-k_{s}^{*} t^{*} / K_{e}^{*}} \mathbf{P e}_{T} \mathbf{D} \mathbf{a}_{T}, \\
c_{s}^{e f f}\left(x^{*}, t^{*}\right)=c_{s 0}^{*} e^{-k_{s}^{*} t^{*} / K_{e}^{*}}+T_{L} k_{s}^{*} \int_{0}^{t^{*}} e^{-k_{s}^{*}\left(t^{*}-\xi\right) / K_{e}^{*}} c_{f u l l}^{e f f}\left(x^{*}, \xi\right) d \xi .
\end{gathered}
$$

and it gives an approximation of order $O\left(\left(\varepsilon \mathbf{P e}_{T}\right)^{3 / 2}\right)$ of $c^{*}$ and $c_{s}^{*}$. More precisely, the approximation is justified rigorously by the following error estimate.

Proposition 1. Let $1 \leq \alpha<2$ and assume that the times scales $T_{A}, T_{D E}$ and $T_{L}$ are of the same order (not depending on $\varepsilon$ ). Assume that $\left(c_{00}^{*}, c_{s 0}^{*}\right) \in$ $\left(C_{0}^{\infty}(\mathbb{R})\right)^{2}$. Let $c^{\text {eff }} \in H^{1}\left(\mathbb{R}_{+} ; H^{2}(\mathbb{R})\right)$ the solution for $(9)-(10)$. Then we have

$$
\begin{gathered}
\left\|c^{*}-c_{f u l l}^{\text {eff }}\right\|_{L^{2}\left(\Omega^{*} \times(0, T)\right)}+\left\|c_{s}^{*}-c_{s}^{e f f}\right\|_{L^{2}\left(\Gamma^{*} \times(0, T)\right)} \\
\leq C\left(\left(\varepsilon \mathbf{P e}_{T}\right)^{3 / 2}\right)\left(\left\|c_{00}^{*}\right\|_{H^{3}(\mathbb{R})}+\left\|c_{s 0}^{*}\right\|_{H^{2}(\mathbb{R})}\right) .
\end{gathered}
$$

On the one hand, in the first line of the effective equation (9), we easily recognize Taylor's model of dispersion. On the other hand, adsorption/desorption reactions introduce complex retardation and memory terms. Some characteristic parameters based on the data from Taylor's article [40] are given in the next section. Let us here mention briefly two important Taylor's examples, one with $\alpha=1.614, H=2.6 \cdot 10^{-4} \mathrm{~m}$, the longitudinal Péclet number $\mathbf{P e}=0.95 \cdot 10^{5}$ and $\mathbf{P e}_{T}=78$ and the second one with $\alpha=1.96, H=2.6 \cdot 10^{-4} \mathrm{~m}$, the longitudinal Péclet number $\mathbf{P e}=4.14 \cdot 10^{6}$ and $\mathbf{P e}_{T}=173$. Obviously, in Taylor's situation, our derived contributions are important and using them is necessary in order to simulate correctly the reactive flows. Since we can not neglect the complex term induced in the model by the kinetics, we give a precise description of their effects in the present paper, through numerical simulations. 


\section{Taylor's example}

All the numerical illustrations of the paper have been computed using the package FreeFem++ [34] and the following space and time discretization:

- Discretization in time: The first order operator is discretized using the method of characteristics. More precisely, a convection equation of the form

$$
\partial_{t} c+\mathbf{q} \cdot \nabla c=f(\mathbf{x}, t)
$$

being given, the one step backward convection scheme by the methods of characteristics reads as follows

$$
\frac{1}{\delta t}\left(c^{n+1}(\mathbf{x})-c^{n}\left(X^{n}(\mathbf{x})\right)=f^{n}(\mathbf{x})\right.
$$

where $c^{n}$ denotes the approximation of the solution $c$ at time $t_{n}=$ $n \delta t$, and $X^{n}(\mathbf{x})$ is an approximation of the solution at time $t_{n}$ of the ordinary differential equation

$$
\frac{d X}{d t}(t)=\mathbf{q}\left(X(t), t_{n}\right), \quad X\left(t_{n+1}\right)=\mathbf{x} .
$$

A Taylor's expansion result implies that $c^{n}\left(\mathbf{x}-\mathbf{q}^{n}(\mathbf{x}) \delta t\right)$ is a first order approximation of $c^{n}\left(X^{n}(\mathbf{x})\right)$. We get the one step backward convection scheme reading:

$$
\frac{1}{\delta t}\left(c^{n+1}(\mathbf{x})-c^{n}\left(\mathbf{x}-\mathbf{q}^{n}(\mathbf{x}) \delta t\right)\right)=f^{n}(\mathbf{x})
$$

Turning back to our complete problem with the diffusion term, we use the following scheme:

$$
\begin{gathered}
\frac{1}{\delta t}\left(c^{n+1}(x, y)-c^{n}(x-q(y) \delta t, y)\right) \\
-\left(D_{x} \partial_{x}^{2}-D_{y} \partial_{y}^{2}\right) c^{n+1}(x, y)=f(x, y, n \delta t) .
\end{gathered}
$$

- Discretization in space: One of the characteristics of our problem is the presence of a smeared front. In order to track it correctly, the Lagrange $P 1$ finite elements with adaptive mesh are used. The mesh is adapted in the neighborhood of front after every ten time steps.

We begin our numerical illustrations by an example issued of the pioneer's work of Taylor [40]. This example is a reference test in view of emphasizing 


\begin{tabular}{|l|l|}
\hline \hline PARAMETERS & VALUES \\
\hline Width of the slit: $H$ & $5 \cdot 10^{-3} \mathrm{~m}$ \\
\hline Characteristic lenght: $L_{R}$ & $0.632 \mathrm{~m}$ \\
\hline$\varepsilon=H / L_{R}$ & $7.9113 \cdot 10^{-3}$ \\
\hline Characteristic velocity: $Q^{*}$ & $3 \cdot 10^{-3} \mathrm{~m} / \mathrm{sec}$ \\
\hline Diffusion coefficient: $D^{*}$ & $2 \cdot 10^{-7} \mathrm{~m}^{2} / \mathrm{sec}$, \\
\hline Longitudinal Peclet number: $\mathbf{P e}=L_{R} Q^{*} / D^{*}=$ & $9.48 \cdot 10^{3}$ \\
\hline$\alpha=\log \mathbf{P e} / \log (1 / \varepsilon)=$ & 1.8921440 \\
\hline Transversal Peclet number: $\mathbf{P e}_{T}=H Q^{*} / D^{*}=$ & 75 \\
\hline \hline
\end{tabular}

Table 1: Parameters values in Taylor's paper

the differences between a transport without kinetical effects and a transport under order one Damköhler number as presented in the next sections. We compute the following set of parameters thanks to the precise description by Taylor of his experimental setup and obtain Table 1 . Note that we choose here the critical example from [40] where $\alpha$ is very close to the threshold value $\alpha^{*}=2$.

For this set of parameters, we compute the vertical average of the solution $c^{*}$ of equation (1), and we compare it with the solution $c^{\text {Tay }}$ of (4). In order to show that in this situation one can not neglect Taylor's dispersive contribution, we also compute the solution $c^{\text {moy }}$ of a very brute approximation by simple averaging, that is

$$
\partial_{t^{*}} c^{m o y}+\frac{2 Q^{*}}{3} \partial_{x^{*}} c^{m o y}-D^{*} \partial_{x^{*}}^{2} c^{m o y}=0 \quad \text { in } \mathbb{R}_{+} .
$$

For the three equations, we choose the following initial and left boundary conditions:

$$
c_{\mid x=0}=1, \quad c_{\mid t=0}=0 .
$$

Originally this problem is formulated in a semi-infinite channel. In our numerical computations we have considered a finite one of length $N L_{R}, N \in$ $\mathbb{N}^{*}$ being chosen sufficiently large for our purpose. This prevents the outflow boundary condition to influence the tracer breakthrough at the observation points. For instance, setting $N=4$ was sufficient for our simulations. At the outflow we have imposed a homogeneous Neumann boundary condition

$$
\partial_{x^{*}} c_{\mid x=N L_{R}}=0 \text {. }
$$

Some results are presented in Figure 1. They show clearly the smallness of the error between exact and Taylor's approximate solution and the advantage of Taylor's model over the model obtained by taking the simple mean over the vertical section. 


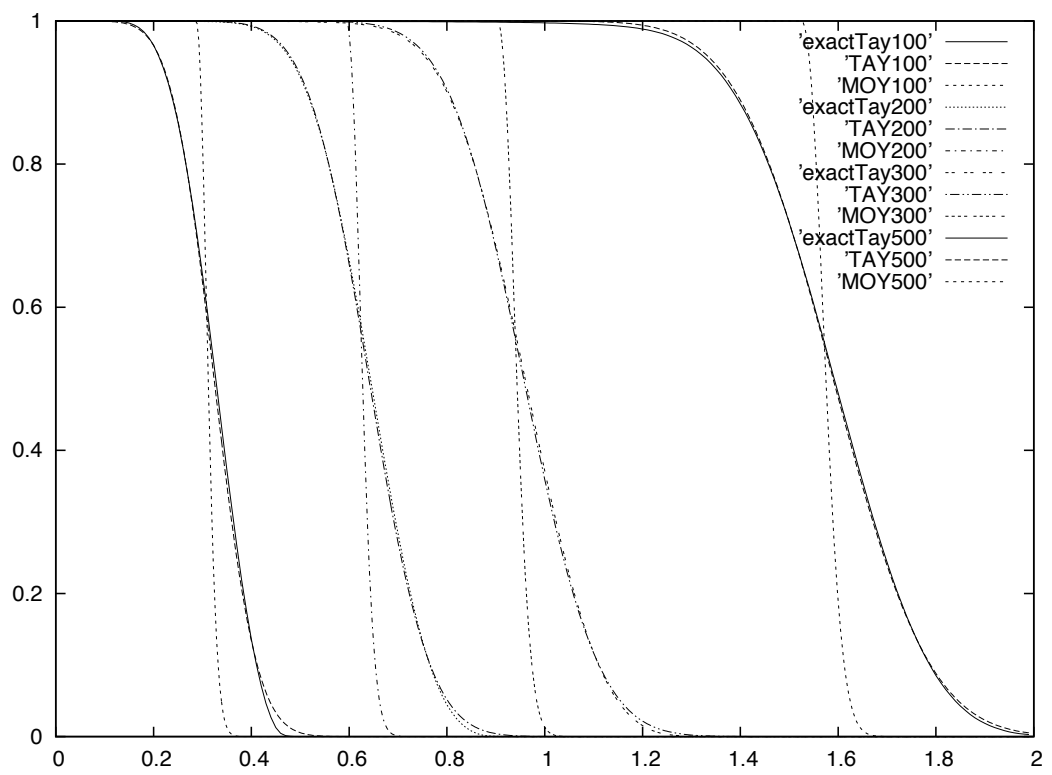

Figure 1: Comparison between concentration from Taylors paper ('tay') and from the simple average ('moy') with the vertically averaged solution of the original problem ('exactTay'), at times $t^{*}=100,200,300,500 \mathrm{sec}$.

Note that here, in absence of chemical reactions, we can solve explicitly the effective problem (4) using the integral formula:

$$
\begin{gathered}
c^{\text {Tay }}(x, t)=1-\frac{1}{\sqrt{\pi}}\left(\exp \left(\frac{\langle q\rangle x}{D^{\text {Tay }}}\right) \int_{(x+\langle q\rangle t) /\left(2 \sqrt{D^{\text {Tay }}}\right)}^{\infty} e^{-\eta^{2}} d \eta\right. \\
\left.+\int_{(x-\langle q\rangle t) /\left(2 \sqrt{D^{T a y} t}\right)}^{\infty} e^{-\eta^{2}} d \eta\right), \quad\langle q\rangle=2 Q^{*} / 3 .
\end{gathered}
$$

An analogous result holds true for the solution of (14). We thus also have used this first setting to calibrate our numerical scheme. As an illustration, we present in Figure 2 the evolution of the variance for small times, computed respectively with our numerical scheme and with the explicit solution (definition of the variance being recalled in the beginning of Subsection 4.2 below). Obviously the numerical results meet well the analytical solution. The detailed analysis of the variance is postponed to Subsection 4.2.

\section{Numerical simulations on the effective model}

This section presents the main results of the paper. We develop numerical simulations for the effective model derived in [15] for Taylor's dispersion 


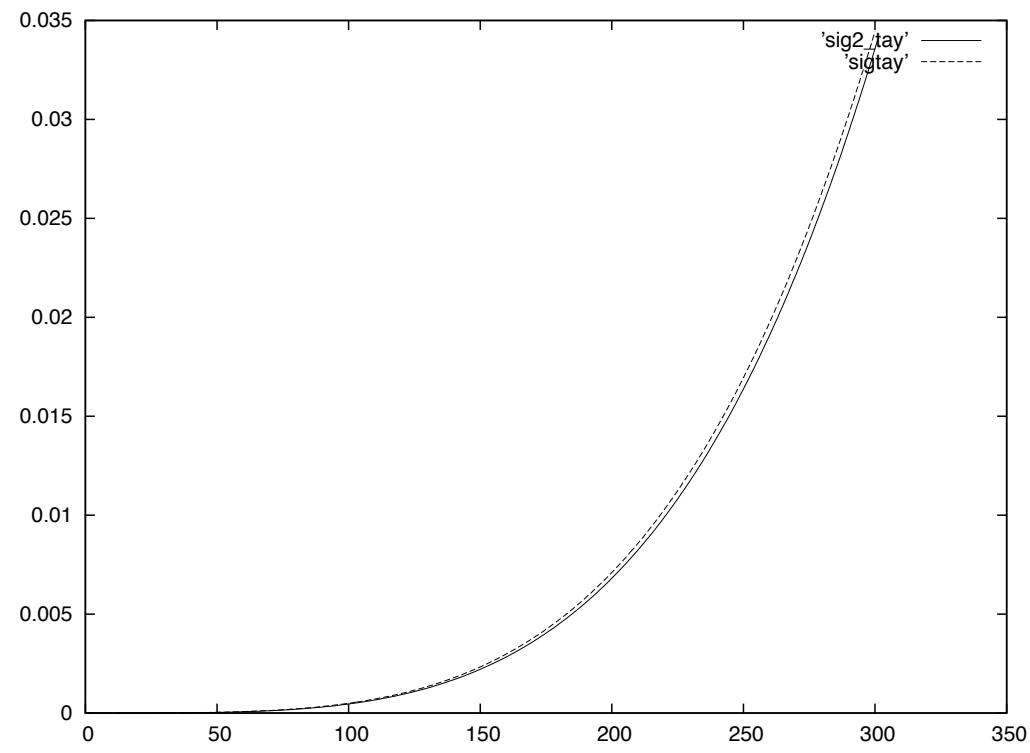

Figure 2: Comparison between variance computed with the analytical solution ('sig2_tay') and with our numerical scheme ('sigtay')

under dominant Damköhler numbers.

\subsection{Comparison of the exact and effective solutions}

Retardation and memory effects on the dispersive characteristics due to the adsorption/desorption reactions appear in the effective model (9)-(12). In the present section, we aim to show their importance. In particular the chemistry influences directly the characteristic diffusion width. We thus perform similar simulations to the ones presented in Section $\S 3$ : we compare now the transverse average of the solution $c^{*}$ of the real problem (6)-(8) with the effective approximation defined by (9)-(11). We use the same set of parameters as in Section $\S 3$ Table 1 with the ones for the reactions characteristics described in Table 2 . We choose $k_{s}^{*}=Q^{*} \varepsilon, K_{e}^{*}=H, T_{A}=T_{L}$.

In presence of the reactions described through (7) and Table 2, the analogous of Figure 1 is Figure 3. Clearly, there is a good agreement between the exact and effective solutions. The small differences are essentially due to the value of parameter $\varepsilon=H / L_{R}$ which is quite important in this example. It is sufficient to divide $\varepsilon$ by 2 (hence diminishing the term $C\left(\left(\varepsilon \mathbf{P e}_{T}\right)^{3 / 2}\right)$ in the error estimate (13)) to drastically reduce the differences (see Figure 4). 


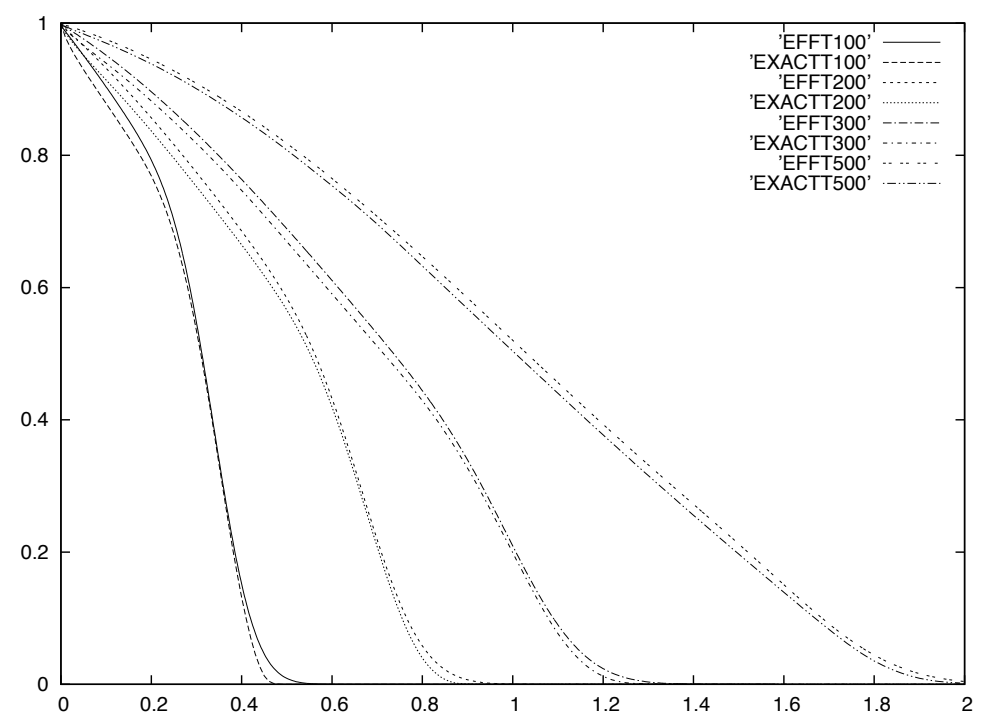

Figure 3: Comparison between concentration from the vertical average of the original problem ('EXACTT') and from the effective model ('EFFT') at times $t^{*}=100,200$, 300,500 sec.

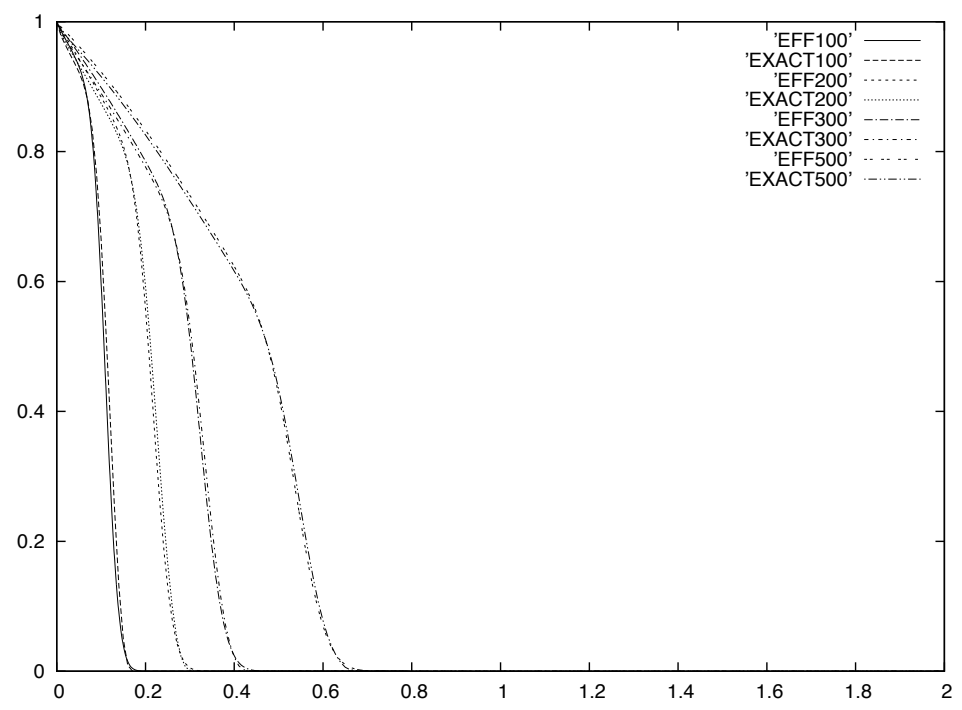

Figure 4: Similar results to those of Figure 3 but computed for a value of $\varepsilon$ divided by 2 


\begin{tabular}{|l|l|}
\hline \hline PARAMETERS & VALUES \\
\hline Adsorption rate constant: $k_{s}^{*}$ & $2.37 \cdot 10^{-5} \mathrm{~m} / \mathrm{sec}$ \\
\hline Adsorption equilibrium constant: $K_{e}^{*}$ & $5 \cdot 10^{-3} \mathrm{~m}$ \\
\hline Longitudinal Damköhler number: $\mathbf{D a}=\frac{L_{R}}{T_{A} Q^{*}}=$ & 1 \\
\hline Transversal Damköhler number: $\mathbf{D a} a_{T}=\frac{k_{s}^{*}}{Q^{*}}=$ & $7.9113 \cdot 10^{-3}$ \\
\hline \hline
\end{tabular}

Table 2: Parameters values for the kinetics

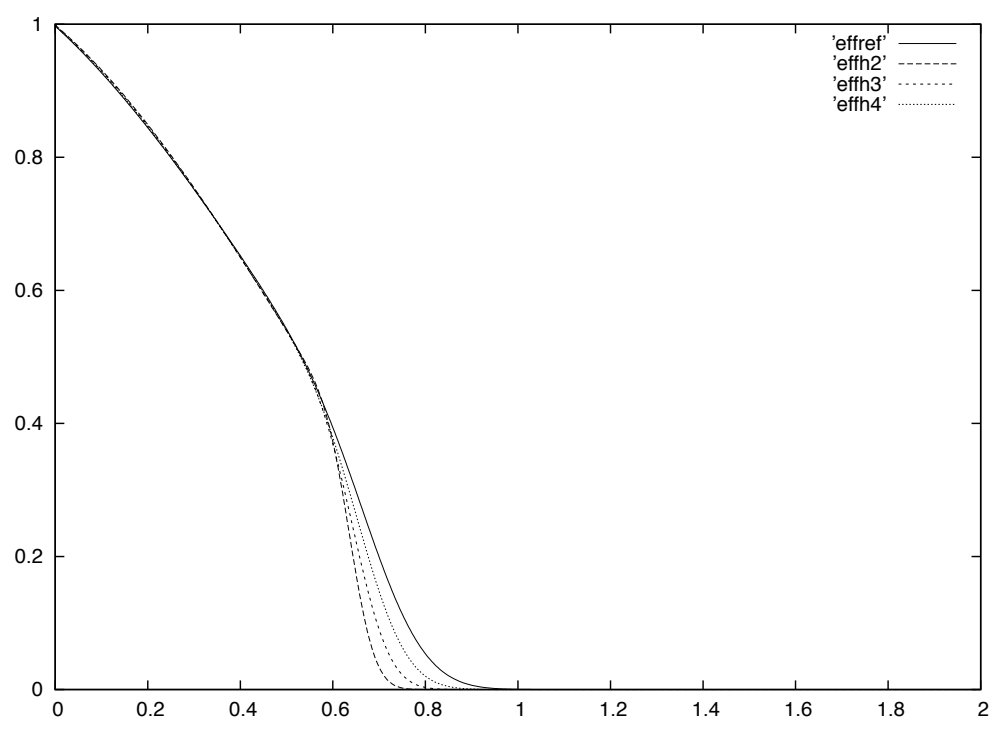

Figure 5: Comparison between the profile of the effective concentration presented in Figure 2 when $H=5 \cdot 10^{-3}$ ('effref') and the one of the effective concentration when $H=N \cdot 10^{-3}$ ('effhN') at time $t^{*}=200 \mathrm{sec}$.

We add some numerical simulations to highlight the kinetics effects on the solute spread in Figures 5, 6, 7 .

In Figure 5, we present a reference example with the profiles obtained as the height $H$ varies. If $H$ decreases, so does the transverse Péclet number $\mathbf{P e}_{T}$ and the effective diffusion coefficient: we observe a global retardation in the spread of the front. A decrease of $H$ also means a decrease of the superficial chemical reaction time scale. One observes its effect at the head of the front.

In Figure 6, we present a reference example with the profiles obtained when the axial velocity $Q^{*}$ varies. A decrease of $Q^{*}$ means an increase of the transverse Damköhler number $\mathbf{D} \mathbf{a}_{T}$. The observed profiles are thus more stiff because more slowed down by a relatively stronger adsorption rate. 


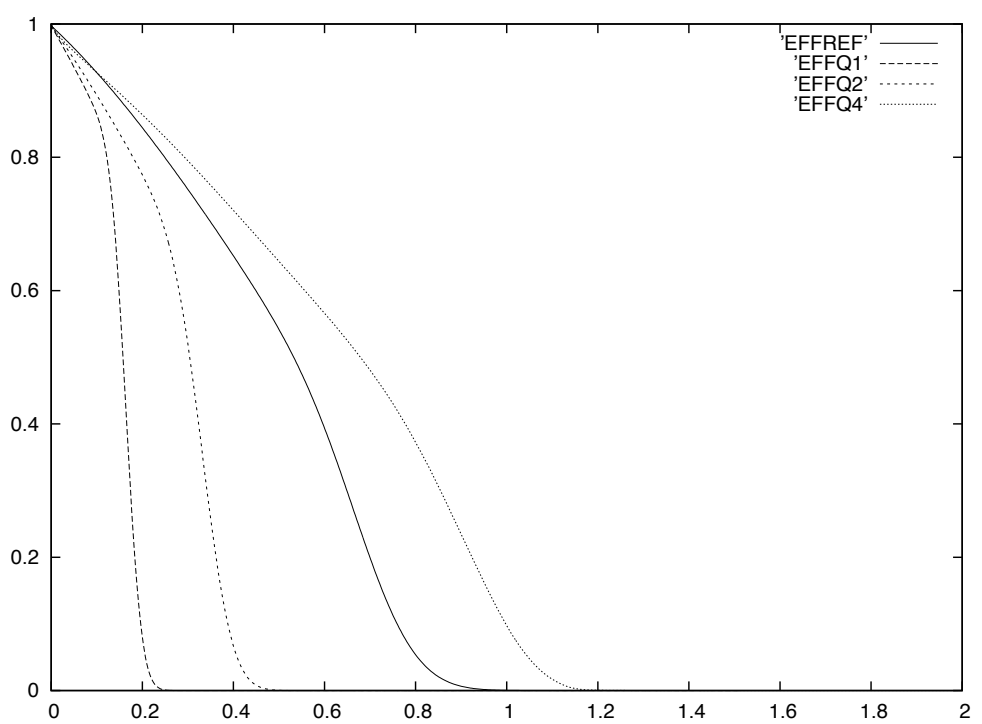

Figure 6: Comparison between the profile of the effective concentration presented in Figure 2 when $Q^{*}=3 \cdot 10^{-3}$ ('EFFREF') and the one of the effective concentration when $Q^{*}=N \cdot 10^{-3}$ ('EFFQN') at time $t^{*}=200 \mathrm{sec}$.

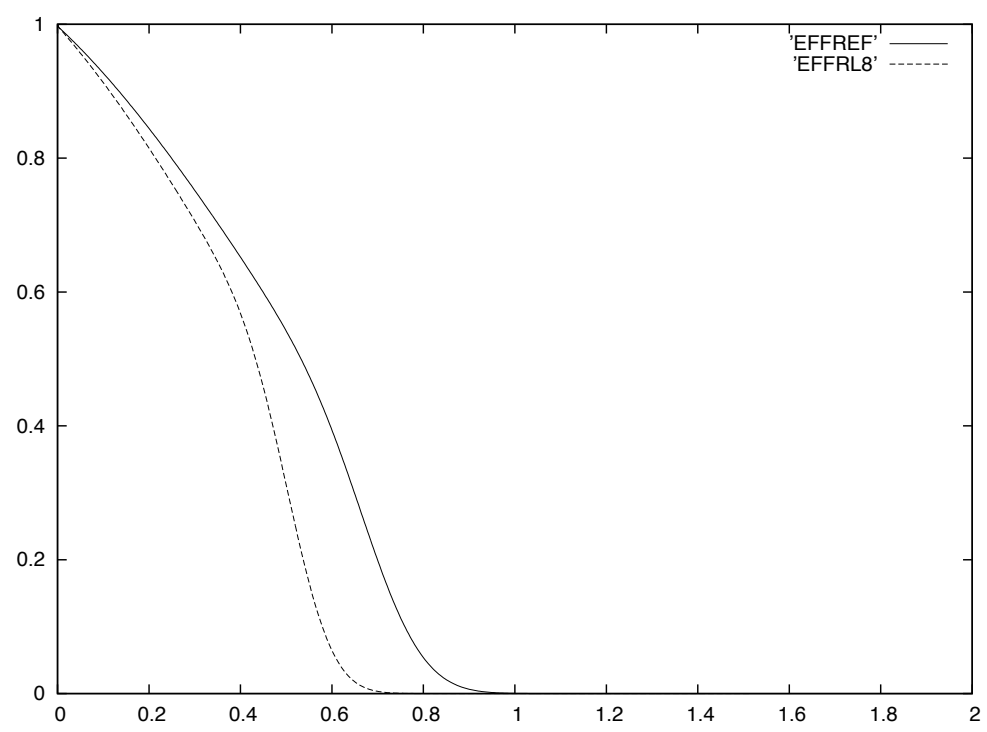

Figure 7: Comparison between the profile of the effective concentration presented in Figure 3 when $L_{R}=0.632$ ('EFFREF') and the one of the effective concentration when $L_{R}=0.832$ ('EFFRL8') at time $t^{*}=200 \mathrm{sec}$. 
Figure 7 presents an example with a decrease of both Péclet and Damköhler numbers. It should induce of course a net slow down of the propagation of the front. This is confirmed by the simulations on the upscaled model (9)-(11) presented in figure 7. Note in particular that with our choice of parameters, the increase of $L_{R}$ induces a decrease of $\varepsilon=\mathbf{D} \mathbf{a}_{T}$ appearing in (9)-(11).

\subsection{Enhanced and anomalous diffusion}

We recall that informations on a diffusive regime may be obtained by computing, for a given concentration $c$, the first moments about the origin $M_{k}$ and the associated variance $\sigma^{2}$, defined by

$$
\begin{gathered}
M_{k}(t)=\int_{-\infty}^{+\infty} x^{k} c(x, t) d x, \quad 0 \leq k \leq 2, \\
\sigma(t)^{2}=M_{2}(t) M_{0}(t)-M_{1}(t)^{2} .
\end{gathered}
$$

The characteristic diffusion $D_{c}$ is then such that $\sigma(t) \sim \sqrt{D_{c} t}$. Nevertheless, observations have emphasized the existence of regimes where $\sigma$ rather expands as $t^{\alpha}, \alpha \neq 1 / 2$ (see for instance the observations in a sand column described in [8]). This phenomenon is referred to as anomalous diffusion.

The question of enhanced and anomalous diffusion arises naturally from the fundamental work of Taylor [40]:

- Taylor showed that, for sufficiently large time, any point discharge of tracer in laminar pipe Poiseuille flow evolves to a symmetric gaussian distribution moving longitudinally with the mean speed of the flow

and with a characteristic diffusion width $\sigma \sim \sqrt{D^{*}\left(1+\frac{8}{945} \mathbf{P e}_{T}^{2}\right) t}$. This is an enhanced diffusion compared to the original one $\sigma \sim \sqrt{D^{*} t}$.

- In the mean time, Taylor, in his experiments, noted that for moderate time a distinct asymmetry was observed, in contradiction with a fickian behavior.

By ignoring longitudinal diffusion and interactions with the pipes boundary, Lighthill [26] showed that the tracer distribution spreads longitudinally proportional to $t$ for the moderate time. This is perhaps the first observation of anomalous diffusion in the fluids literature. Then, Chatwin [13] generalized the Aris result for transversely uniform initial data to the full variance temporal evolution, and computed short time asymptotic behavior of this special case. Short times behavior is also the preoccupation of [39]. 
More recently, Latini and Bernoff [25] generalized the method employed by Lighthill to consider the anomalous spread of an initial distribution before complete transverse mixing has occurred. The authors of [12] employ the stochastic differential equations underlying the passive scalar equation and the rules of conditional probability to compute, with no approximations, the moments needed to construct the complete variance valid for all times. The long time limit of the variance is shown to agree with Taylor's type result. References for reactive settings are seldom. Motivated by the study of a flow in a catheterized artery with conductive walls, the authors of [31] considered a similar problem in an annular pipe, but for a very simple model of reaction, i.e. an instantaneous linear equilibrium. They use a Crank-Nicholson scheme coupled with series expansion, once again formal, to approximate the mean concentration distribution using the first four central moments. Let us finally mention the recent work [6] which clearly describes difficulties induced by the modeling of preasymptotic times and which moreover evaluate mixing.

Difficulty is to determine the short-time limit of the domain of applicability of Taylor's type models since they are constructed to hold true at large times. Looking for an answer through numerical simulations was done as soon as some years after Taylor's work (see [21]). We now show that the effective model (9)-(11) is able to capture the three regimes of diffusion encountered in such a problem of tracer injection in a thin pipe:

Diffusive regime Initially, diffusion dominates over advection yielding a spherically symmetric Gaussian dispersion cloud.

Note that in the simplest case of absence of reactions, longitudinal displacement due to diffusion is of order $\mathcal{O}\left(\sqrt{D^{*} t}\right)$. The relative longitudinal displacement of two particles is controled by $\mathcal{O}\left(Q^{*} D^{*} t^{2}\right)$ due to the parabolic shear (compare the convective displacement of a particle at the center of the pipe with the one of a particle that has diffused of $\mathcal{O}\left(\sqrt{D^{*} t}\right)$ in the transverse direction). This regime is then limited to the times $t \ll\left(Q^{* 2} D^{*}\right)^{-1 / 3}$. In the present paper the time limitation also depends on the kinetics.

Anomalous regime In this regime the displacement due to tracer diffusing transversely and being sheared longitudinally dominates over the longitudinal diffusion. In this regime, the variance show an anomalous diffusion and the distribution of solute concentration is distinctly asymmetric.

As emphasized by Latini and Bernoff [25], this kind of phenomenon appears when the majority of the tracer has not yet interacted with 
the pipes boundary. Here, in the case of reacting wall, the effects are enhanced.

Taylor's regime At large times, the flow is in the classical Taylor regime, for which the tracer is homogenized transversely across the pipe and diffuses with a Gaussian distribution longitudinally.

In the original setting of Taylor (see equations (4)-(5)), the diffusion is enhanced by the factor $8 D^{*} \mathbf{P e}_{T}^{2} / 945$. In the present paper, there is a greater enhancement due to the reactions.

Let us illustrate that the three latter behaviors are captured by model (9)-(11). Due to the complex structure of the effective equation (9), the observation of these latter phenomena is not obvious. Numerical simulations thus become a particularly interesting tool.

To deal with large times computations, we slightly modify our set of parameters. Tables 1, 2 are thus replaced by Table 3 . The tracer is initially injected until $t_{0}=4000 \mathrm{sec}$.

\begin{tabular}{|l|l|}
\hline \hline PARAMETERS & VALUES \\
\hline Width of the slit: $H$ & $10^{-1} \mathrm{~m}$ \\
\hline Characteristic lenght: $L_{R}$ & $1000 \mathrm{~m}$ \\
\hline$\varepsilon=H / L_{R}$ & $10^{-4}$ \\
\hline Characteristic velocity: $Q^{*}$ & $9 \cdot 10^{-4} \mathrm{~m} / \mathrm{sec}$ \\
\hline Diffusion coefficient: $D^{*}$ & $2 \cdot 10^{-7} \mathrm{~m}^{2} / \mathrm{sec}$, \\
\hline Longitudinal Peclet number: Pe $=L_{R} Q^{*} / D^{*}=$ & $4.5 \cdot 10^{6}$ \\
\hline$\alpha=\log \mathbf{P e} / \log (1 / \varepsilon)=$ & 1.6633 \\
\hline Transversal Peclet number: $\mathbf{P e}_{T}=H Q^{*} / D^{*}=$ & 450 \\
\hline Adsorption rate constant: $k_{s}^{*}$ & $9 \cdot 10^{-8} \mathrm{~m} / \mathrm{sec}$ \\
\hline Adsorption equilibrium constant: $K_{e}^{*}$ & $5 \cdot 10^{-1} \mathrm{~m}$ \\
\hline Longitudinal Damköhler number: $\mathbf{D a}=\frac{L_{R}}{T_{A} Q^{*}}=$ & 1 \\
\hline Transversal Damköhler number: $\mathbf{D a} \mathbf{a}_{T}=\frac{k_{s}^{*}}{Q^{*}}=$ & $10^{-4}$ \\
\hline \hline
\end{tabular}

Table 3: Parameters values for the variances computations

We begin by computing the evolution of the variance associated to the upscaled reactive problem (9)-(11). The result is presented in Figure 8. Observation of the evolution of the variance let us detect the three different regimes of diffusion:

- For $t^{*} \ll 2.5 \cdot 10^{5}$, the fluid obeys to the classical diffusive regime, with the diffusion coefficient $D^{*}$ (actually, the diffusion $D^{*}$ is so small here that it is difficult to differentiate the line of slope $D^{*}$ of the horizontal axis). 


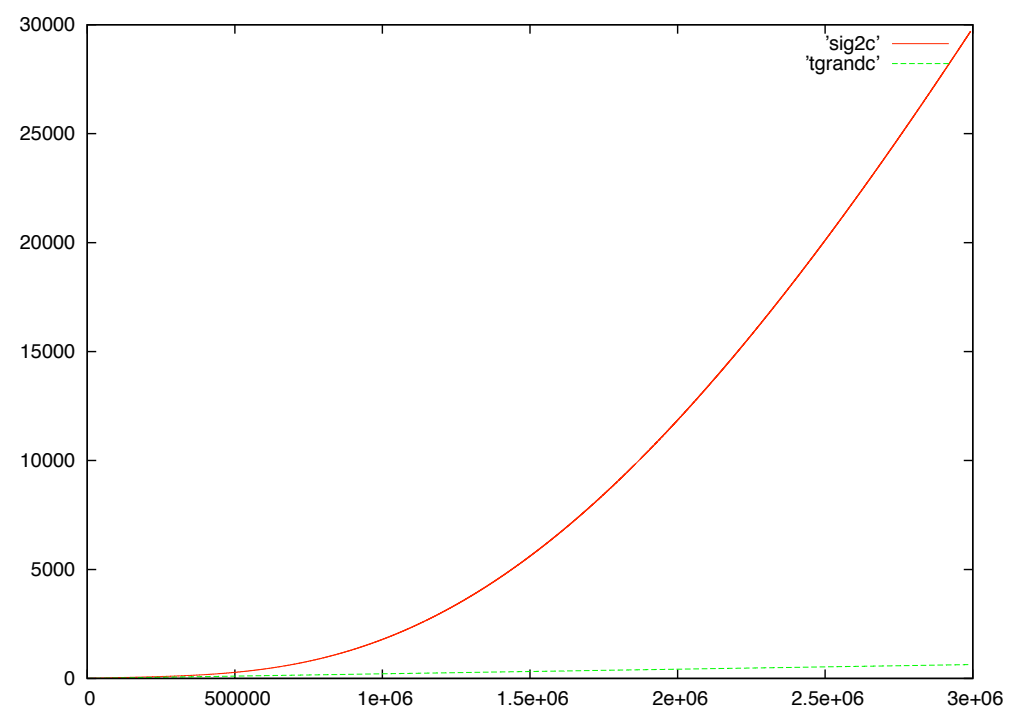

Figure 8: Evolution of the variance (with respect to time) for the reactive problem

- Then, for $2.5 \cdot 10^{5} \ll t^{*}$, we clearly observe a zone of anomalous regime where $\sigma^{2}(t) \nsim C t, C \in \mathbb{R}$, followed by a return to a diffusive regime.

The point is to detect the transition times from anomalous to enhanced diffusion. A method, e.g. used in [31], consists in observing eventual asymmetry in the concentrations profiles. Some of them, computed during the anomalous period, are presented in Figure 9. Nevertheless this method is obviously not practicable neither confident at large times.

Observation of anomalous regime should be confirmed by the computation of the skewness $S k$, defined by

$$
S k(t)=\frac{M_{0}(t)^{2} M_{3}(t)-3 M_{0}(t) M_{1}(t) M_{2}(t)+2 M_{1}(t)^{3}}{\sigma^{3}(t)} .
$$

The skewness is an indicator of the symmetry of the concentration's distribution. Results are presented in Figure 10. The negative value of the skewness indicates an asymmetric profile with a left tail more pronounced than the right tail (as in Figure 9). Computation of the skewness evolution seems to be the right tool to assert that fickian behavior can only be supposed for $10^{7}$ sec. $\ll t^{*}$ :

- For $t^{*} \gg 10^{7} \mathrm{sec}$. (see Figure 11), we turn back to a diffusive regime, actually a Taylor's type enhanced diffusion regime: the value of the effective diffusion is given by the slope of the tangent to the curve of the squared 


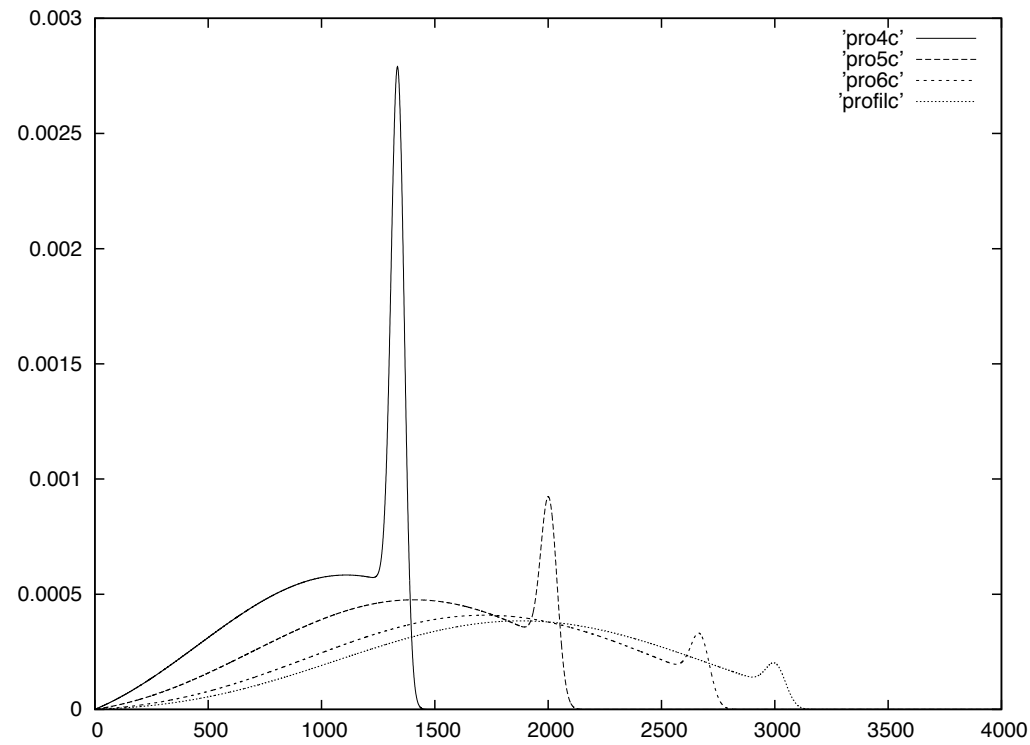

Figure 9: Asymmetric profiles (with respect to space) during the period of anomalous diffusion at times $t^{*}=4 \cdot 10^{6}$ sec., $5 \cdot 10^{6}$ sec., $6 \cdot 10^{6}$ sec. and $6.5 \cdot 10^{6} \mathrm{sec}$.

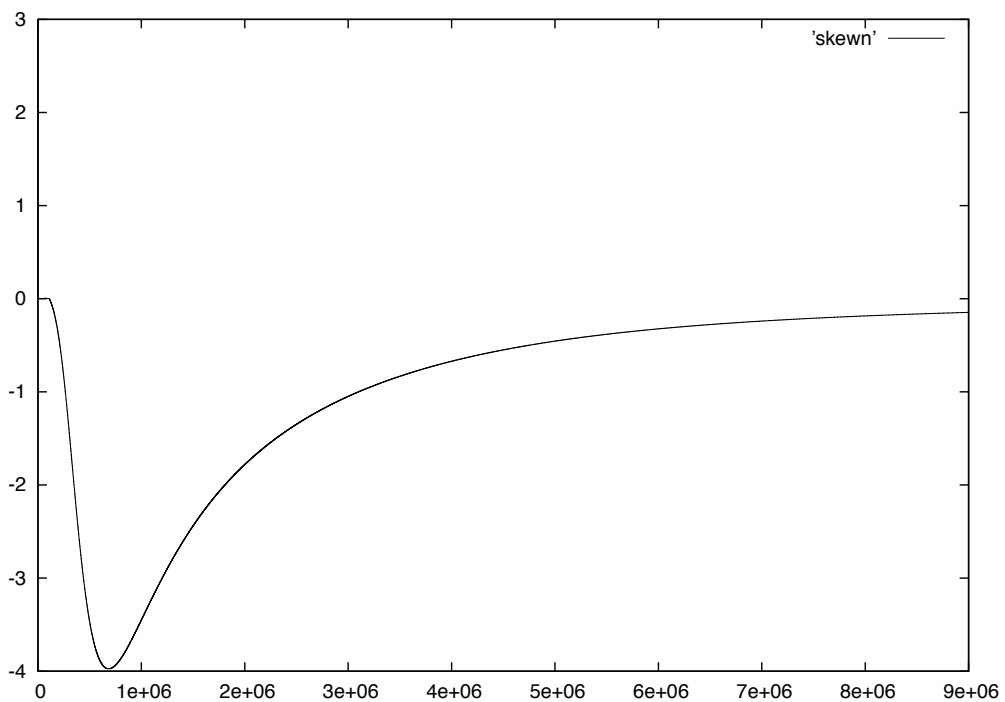

Figure 10: Evolution of the skewness corresponding to Figure 8 


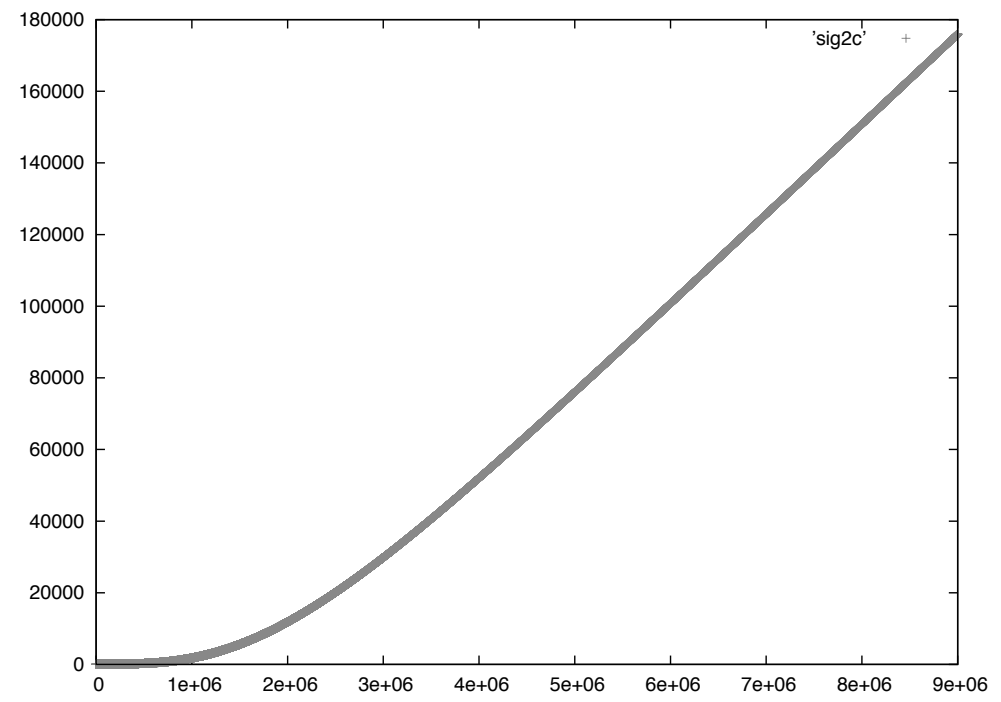

Figure 11: Evolution of the variance for the reactive problem at larger times

variance.

We note easily that Taylor's enhanced diffusion is drastically increased by the reactive effects by comparing the slope of the asymptotes at large times of variance curves for reactive and Taylor's settings (Figures 11 and 12 respectively): in Figure 11, a line of slope $D^{\text {Tay }}$ would be hard to distinct from an horizontal line. In Figure 8 (respectively Fig. 12) line of slop $D^{\text {Tay }}$ is 'tgrandc' (respectively 'tgrandtt').

Furthermore, by comparing figures 11 and 12, we check straightforward that Taylor's regime of enhanced diffusion appears much later in presence of reactions. Kinetics make the anomalous diffusion regime longer.

\subsection{Effects of the kinetics on the convection}

In the former subsection, we have shown that the effective model, despite being derived from the conventional diffusive Fick law at the microscopic level, exhibits anomalous and enhanced diffusive effects. Moreover, the effect of boundary adsorption increases the deviation from the Gaussian distribution.

Now, we aim to emphasize that the reactions also have influence on the apparent mean velocity of the flow. We thus introduce the following equation, which is equation (9) where we have suppressed all the retardation 


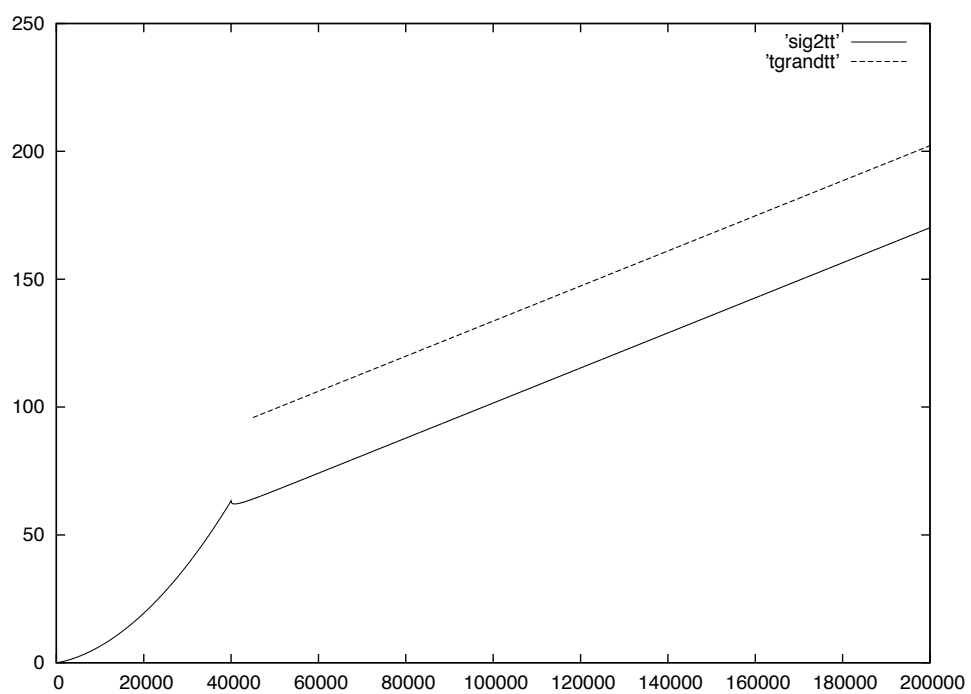

Figure 12: Evolution of the variance corresponding to Taylor's setting (without reactions). The straight line has a slope of value $D^{\text {Tay }}, D^{\text {Tay }}$ being the value of the enhanced diffusion during the second fickian regime as $t^{*} \gg 4 \cdot 10^{4}$. The first fickian regime, characterized by the diffusion $D^{*}$, is detectable by zooming to smaller times $t^{*}<D^{-1 / 3} \approx 30$ sec. 
terms which act on convection:

$$
\begin{gathered}
\frac{\partial c^{g l o b}}{\partial t^{*}}+\frac{2}{3} Q^{*} \frac{\partial c^{g l o b}}{\partial x^{*}}-D^{*}\left(1+\frac{8}{945} \mathbf{P e}_{T}^{2}\right) \frac{\partial^{2} c^{g l o b}}{\partial\left(x^{*}\right)^{2}} \\
+\frac{k_{s}^{*}}{K_{e}^{*}} c^{g l o b}-\left(\frac{k_{s}^{*}}{K_{e}^{*}}\right)^{2} \int_{0}^{t^{*}} e^{-k_{s}^{*}\left(t^{*}-\xi\right) / K_{e}^{*}} c^{g l o b}(\cdot, \xi) d \xi \\
-\frac{1}{3} \mathbf{P e}_{T} \mathbf{D} \mathbf{a}_{T} \frac{H}{K_{e}^{*}} \frac{k_{s}^{*}}{K_{e}^{*}}\left(c^{g l o b}\right. \\
\left.+\int_{0}^{t^{*}} e^{-k_{s}^{*}\left(t^{*}-\xi\right) / K_{e}^{*}}\left(\left(\frac{k_{s}^{*}}{K_{e}^{*}}\right)^{2}\left(t^{*}-\xi\right)-2 \frac{k_{s}^{*}}{K_{e}^{*}}\right) c^{g l o b}(\cdot, \xi) d \xi\right) \\
=\frac{k_{s}^{*}}{K_{e}^{*}} e^{-k_{s}^{*} t^{*} / K_{e}^{*}}\left(\frac{c_{s 0}^{*}}{K_{e}^{*}}+\frac{1}{3} \mathbf{P e}_{T} \mathbf{D} \mathbf{a}_{T} \frac{c_{s 0}^{*}}{K_{e}^{*}} \frac{H}{K_{e}^{*}}\left(\frac{k_{s}^{*}}{K_{e}^{*}} t^{*}-1\right)\right) \\
+e^{-k_{s}^{*} t^{*} / K_{e}^{*}} \frac{4}{45} \frac{k_{s}^{*}}{K_{e}^{*}} \frac{H}{K_{e}^{*}} \mathbf{P e}_{T} \partial_{x} c_{s 0}^{*},
\end{gathered}
$$

We then compare the solutions issued of (9) with the ones issued of (15) and of Taylor's model without kinetics (4). Computations performed at $t^{*}=200$ and $t^{*}=1000,1400$ are presented in Figures 13-15. First, for small times, the effective convectional velocity seems to slightly increase (see the retard of curve 'glo' with regard to curve 'eff' in Figure 13). But as of $t^{*}=1000$, we observe easily in Figure 14 the retard of curve 'eff' with regard to the curve 'glo', except at the front of the profile. We also provide the curves corresponding to Taylor's situation 'tay' without reactions to compare this effect with the one due to reactions. This phenomenon worsens for larger times. It means that the wall reactions globally slow down the mean flow of the solute.

\section{Other examples of effective models}

We present finally numerical illustrations for some other effective models: the one derived rigorously in [14] for Danckwerts boundary condition in subsection 5.1, and the one for nonlinear reactions of [18] in subsection 5.2. All the results presented below aim to show the efficiency of the effective models. They show the smallness of the error between exact and effective approximate solution and the advantage of effective models over the models obtained by taking the simple mean over the vertical section.

\subsection{Danckwerts boundary condition}

We assume now an infinite adsorption rate : $k_{s}^{*}=+\infty$ in $(7)$, that is $-D^{*} \partial_{y^{*}} c^{*}=K_{e} \partial_{t} c^{*}$ on $\Gamma^{*}$. At the inlet boundary we suppose Danckwert's 


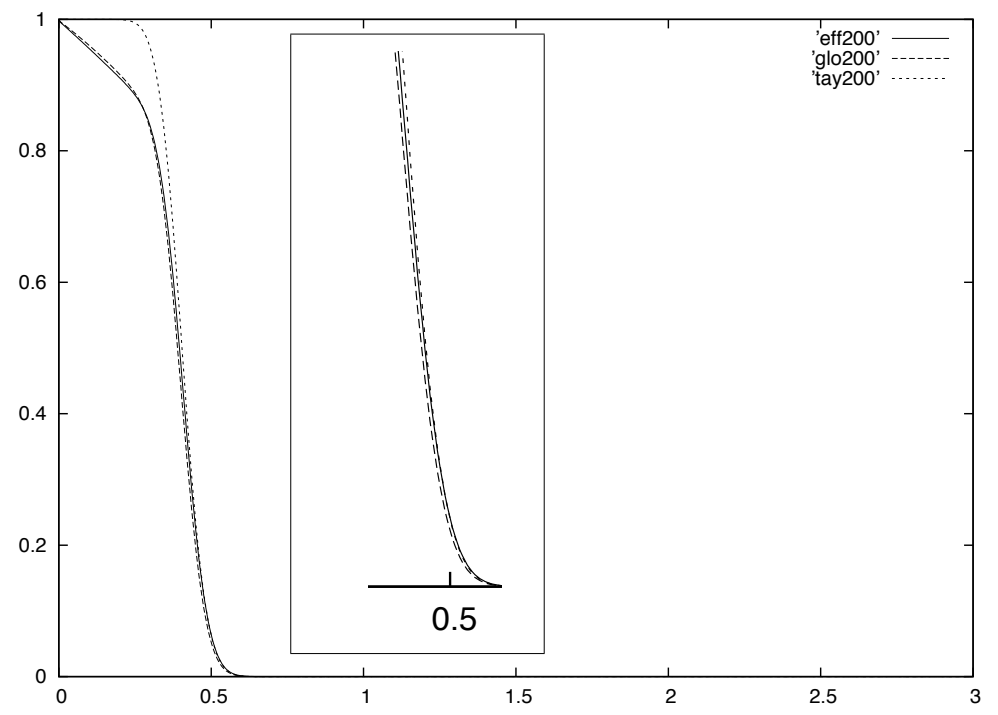

Figure 13: Comparison (with a boxed zoom) between the profiles of solutions issued of the effective model (9) ('eff200') with the ones issued of (15) ('glo200') and of Taylor's model (4) ('tay200') at time $t^{*}=200 \mathrm{sec}$.

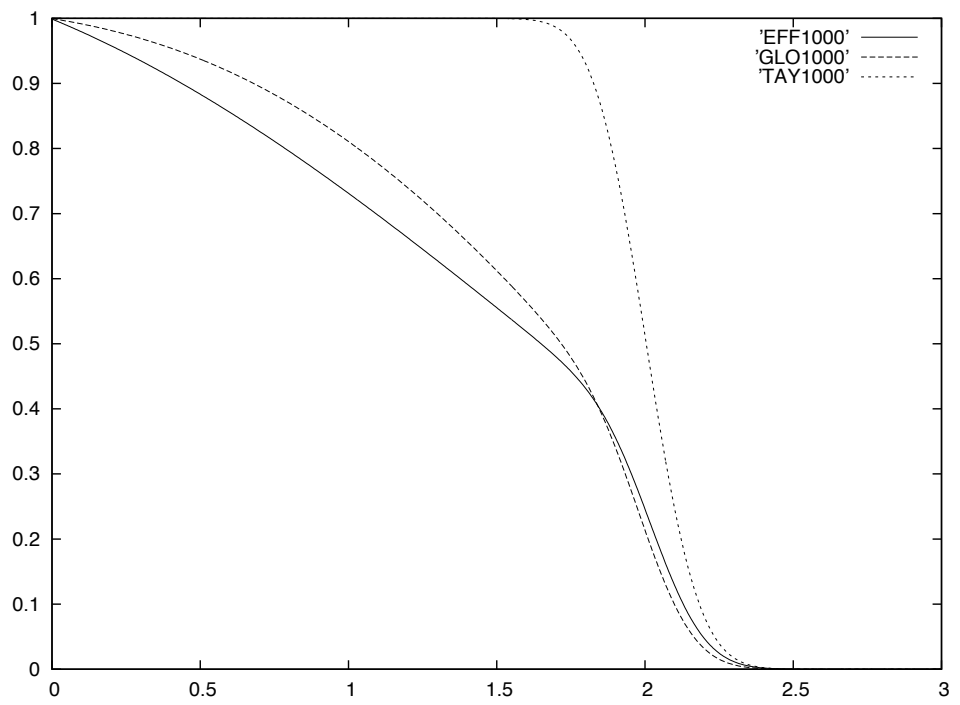

Figure 14: Comparison between the profiles of solutions issued of the effective model (9) ('EFF1000') with the ones issued of (15) ('GL01000') and of Taylor's model (4) ('TAY1000') at time $t^{*}=1000 \mathrm{sec}$. 


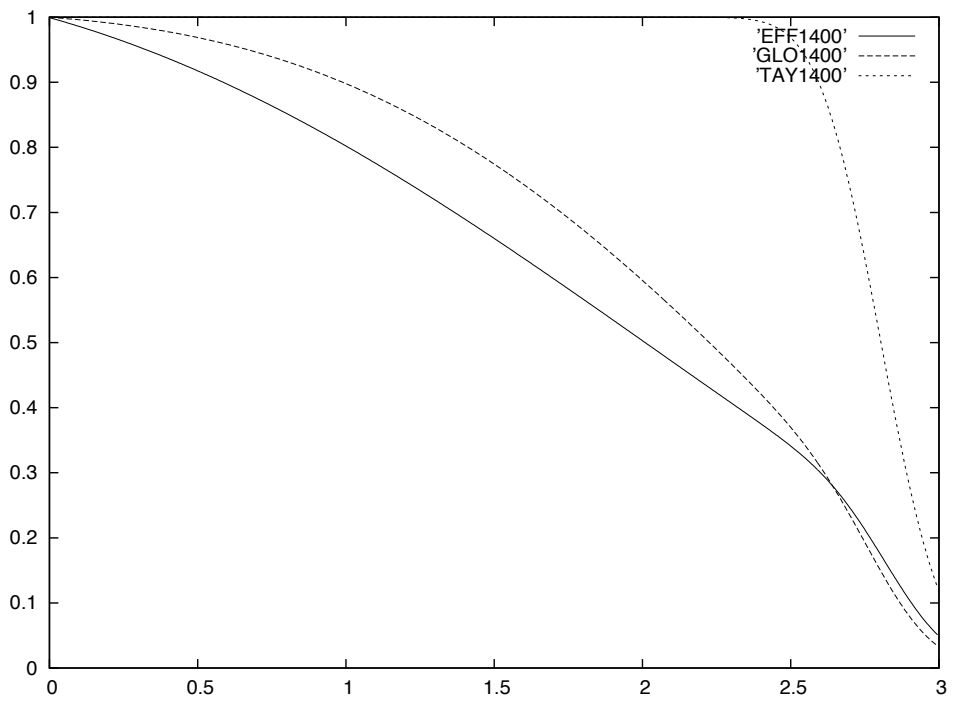

Figure 15: Comparison between the profiles of solutions issued of the effective model (9) ('EFF1400') with the ones issued of (15) ('GL01400') and of Taylor's model (4) ('TAY1400') at time $t^{*}=1400 \mathrm{sec}$.

boundary condition. We have an infiltration with a pulse of water containing a solute of concentration $c_{f}^{*}$ followed by solute-free water. Then the Danckwerts boundary condition at $x^{*}=0$ is:

$$
\begin{gathered}
-D^{*} \partial_{x^{*}} c^{*}+q\left(y^{*}\right) c^{*}=q\left(y^{*}\right) c_{f}^{*} \quad \text { for } \quad 0<t^{*}<t_{0}, \\
-D^{*} \partial_{x^{*}} c^{*}+q\left(y^{*}\right) c^{*}=0 \quad \text { for } \quad t^{*}>t_{0} .
\end{gathered}
$$

The corresponding effective problem is (see its rigorous derivation in [14]):

$$
\begin{gathered}
\left(1+\mathbf{D} \mathbf{a}_{K}\right) \frac{\partial c_{K}^{*, e f f}}{\partial t^{*}}+\frac{2 Q^{*}}{3} \frac{\partial c_{K}^{*, e f f}}{\partial x^{*}}= \\
D^{*}\left(1+\frac{4}{135} \mathbf{P e}_{T}^{2}\left[\frac{2}{7}+\frac{\mathbf{D} \mathbf{a}_{K}\left(2+7 \mathbf{D} \mathbf{a}_{K}\right)}{\left(1+\mathbf{D} \mathbf{a}_{K}\right)^{2}}\right]\right) \frac{\partial^{2} c_{K}^{*, e f f}}{\partial\left(x^{*}\right)^{2}}
\end{gathered}
$$

with $\mathbf{D} \mathbf{a}_{K}=K_{e} / H$, completed by the initial and boundary conditions

$$
\begin{gathered}
-D^{*} \partial_{x^{*}} c_{K}^{*, e f f}{ }_{\mid x=0}+\frac{2 Q^{*}}{3}\left(c_{K}^{*, e f f}{ }_{\mid x=0}-c_{f}^{*} \chi_{\left(0, t_{0}\right)}\right)=0, \\
c_{K}^{*, e f f}{ }_{\mid t=0}=c_{00}^{*} .
\end{gathered}
$$

For the simulations presented in Figures 16-17, we take $c_{f}^{*}=0.5, c_{00}=0$, $\alpha=1.73, \varepsilon=0.001$ and we test successively with $t_{0}=1$ and $t_{0}=0.2$. 


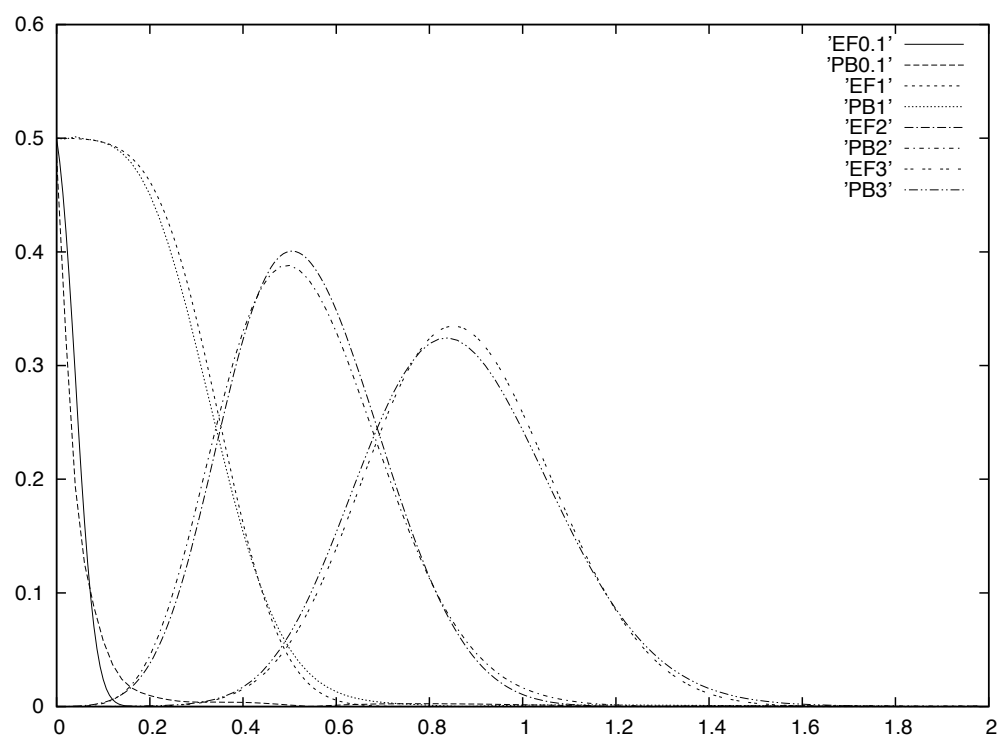

Figure 16: Comparison at times $t^{*}=0.1,1,2,3$ of the effective and exact solutions corresponding to a Danckwerts left boundary condition with $t_{0}=1$ and an infinite adsorption rate

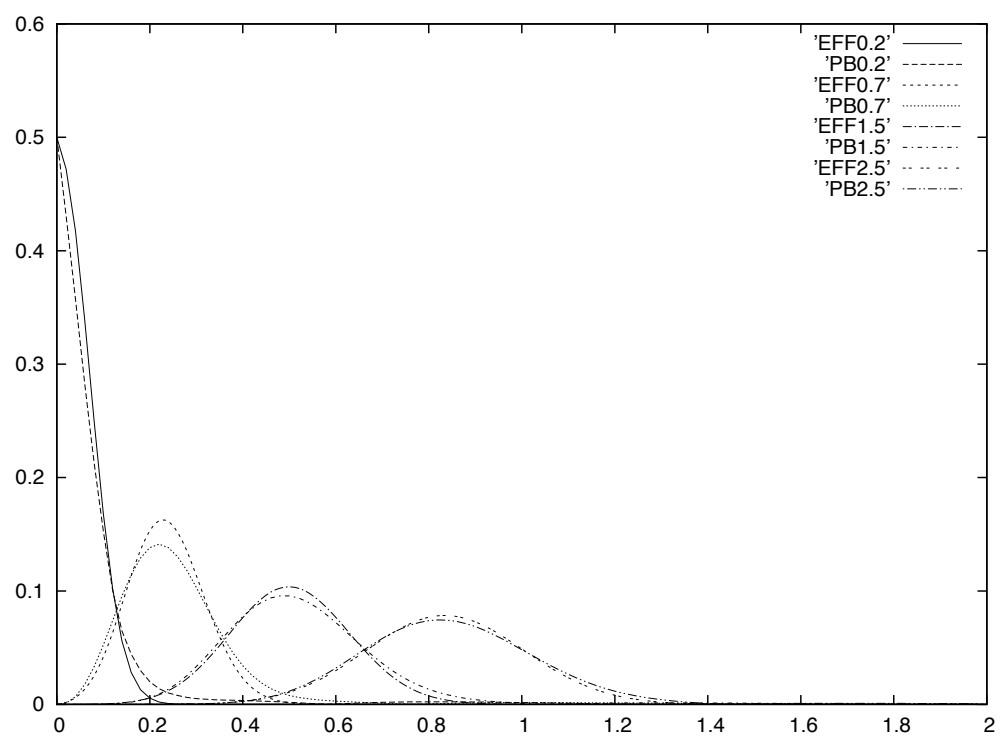

Figure 17: Comparison at times $t^{*}=0.1,0.7,1.5,2.5$ of the effective and exact solutions corresponding to a Danckwerts left boundary condition with $t_{0}=0.2$ and an infinite adsorption rate 


\subsection{Nonlinear kinetics}

Finally we consider some nonlinear models of reactions. Thus Condition (7) is replaced by

$$
-D^{*} \partial_{y^{*}} c^{*}=\frac{\partial c_{s}^{*}}{\partial t^{*}}=\hat{k}_{s}^{*}\left(\Phi\left(c^{*}\right)-c_{s}^{*} / K_{e}^{*}\right) \quad \text { on } \Gamma^{*},
$$

where $\Phi$ is the isotherm function. Typical examples for $\Phi$ are given by the Langmuir and Freundlich isotherms:

$$
\Phi(c)=\frac{k_{1} c}{1+k_{2} c} \quad \text { (Langmuir); } \quad \Phi(c)=k_{1} c^{k_{2}} \quad \text { (Freundlich). }
$$

In the present work, we fix $k_{1}=1$ and use different values for $k_{2}$.

Choosing the same scalings than in the previous sections, the authors of [18] have derived formally (using an anisotropic perturabation method) the corresponding effective model. It reads:

$$
\begin{gathered}
\partial_{t^{*}}\left(c_{N}^{*}+\frac{c_{s, N}^{*}}{H}\right)+\partial_{x^{*}}\left(\frac{2 Q^{*}}{3} c_{N}^{*}+\frac{\mathbf{P e}_{T}}{15} \Phi\left(c_{N}^{*}\right)\right) \\
=D^{*}\left(1+\frac{8}{945} \mathbf{P e}_{T}^{2}\right) \partial_{x^{*}}^{2} c_{N}^{*}+\frac{2 k_{d}^{*} \mathbf{P e}_{T}}{45} \partial_{x^{*}} c_{s, N}^{*} \\
\partial_{t^{*}} c_{s, N}^{*}=\Phi\left(c_{N}^{*}+\mathbf{P e}_{T} \tilde{c}_{N}^{1}\right)-k_{d}^{*} c_{s, N}^{*} \\
\tilde{c}_{N}^{1}=\frac{2 H}{45} \partial_{x^{*}} c_{N}^{*}-\frac{1}{3}, \quad k_{d}^{*}=\frac{k_{s}^{*}}{K_{e}^{*}} .
\end{gathered}
$$

Problem (21)-(23) is thus the equivalent of equation (9) in case of nonlinear reactions. The main difference is due to the nonlinearity of the definition of the reaction which does not allow to decouple the problem for $c^{*}$ from the problem for $c_{s}^{*}$. We thus have to consider here a system of two coupled pde's. Other difference lies in the justification of the effective model (21)-(23). Article [18] contains its formal derivation but no error estimates. For the numerical simulations, we fix $k_{s}^{*}=1, K_{e}^{*}=\frac{H}{Q^{*} \cdot \varepsilon}$. The physical parameters are those already given in the table 1 and we choose the following initial and left boundary conditions:

$$
c_{\mid x=0}=1, \quad c_{\mid t=0}=0 .
$$

First, we take $k_{2}=3$ with Freundlich's adsorption isotherm. Next, we use the Langmuir's adsorption isotherms for the value $k_{2}=2$. Corresponding results are in Figures 18-19. 


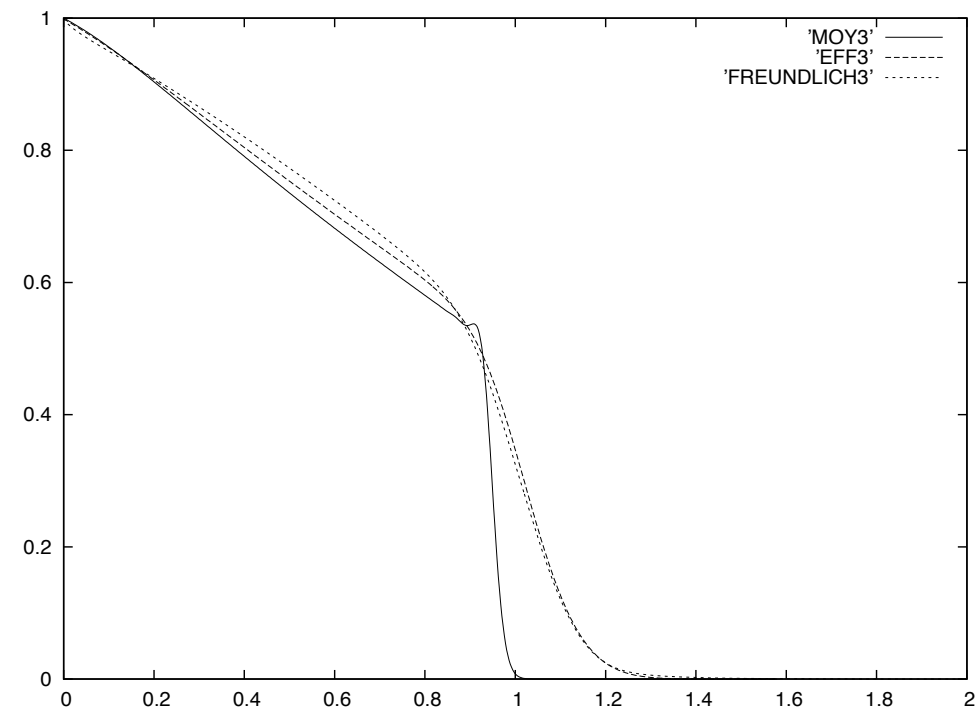

Figure 18: Comparisons between concentrations issued from the effective problem ('eff3'), from the sectional average of the solution of the original problem ('freundlich3') and from the simple average ('moy3'), using Freundlich isotherm characterized by $k_{2}=3$ at time $t=300 \mathrm{sec}$.

\section{Acknowledgment}

Research of the authors was partially supported by the GNR MOMAS (Modélisation Mathématique et Simulations numériques liées aux problèmes de gestion des déchets nucléaires) (PACEN/CNRS, ANDRA, BRGM, CEA, EDF, IRSN) as a part of the project "Multiscale methods for the chemistry coupled with transport in a porous medium". The authors would like to thank Pr. A. Mikelić for stimulating discussions on the subject.

\section{References}

[1] R. Aris, On the dispersion of a solute in a fluid flowing through a tube, Proc. Roy. Soc. London Sect A., Vol. 235 (1956), pp. 67-77.

[2] V. Balakotaiah, H.-C. Chang, Dispersion of Chemical Solutes in Chromatographs and Reactors, Phil. Trans. R. Soc. Lond. A, Vol. 351 ( 1995), no. 1695 , pp. 39-75.

[3] N.G. Barton, An asymptotic theory of dispersion of reactive contaminant in parallel flow, Austr. Math. Soc. B, Vol. 25 (1984), pp. 287-310. 


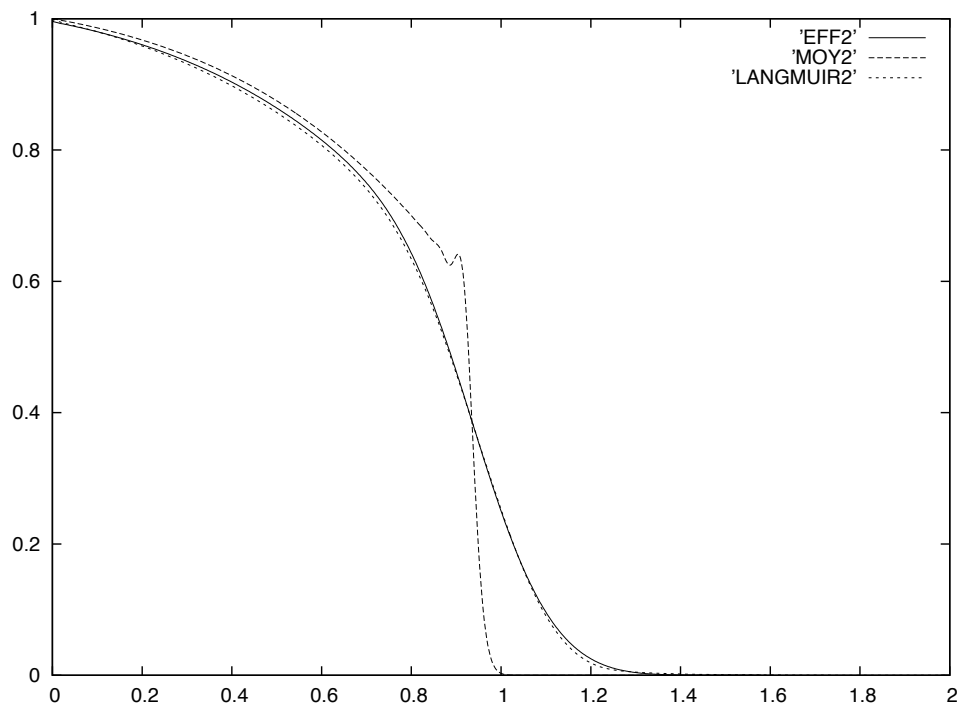

Figure 19: Comparison between concentrations issued from the effective problem ('eff2'), from the sectional average of the solution of the original problem ('langmuir2') and from the simple average ('moy2'), using Langmuir isotherm characterized by $k_{2}=2$ at time $t=300 \mathrm{sec}$.

[4] R.N. Bhattacharya and V.K. Gupta, On the Taylor-Aris theory of solute transport in a capillary, SIAM J. Appl. Math., Vol. 44 (1984), no.1, pp. 33-39.

[5] C.W.J. Berentsen and C.P.J.W. Kruijsdijk, Relaxation and reversibility of extended Taylor dispersion from a Markovian-Lagrangian point of view, Adv. Water Res., Vol. 31 (2008), pp. 609-629.

[6] D. Bolster, F.J. Valdés-Parada, T. LeBorgne, M. Dentz and J. Carrera, Mixing in confined stratified aquifers, J. Cont. Hydr., Vol. 120-121 (2011), pp. 198-212.

[7] H. Brenner, Dispersion resulting from flow through spatially periodic porous media, Phil. Trans. Roy. Soc. Lond. A, Vol. 297 (1980), pp. 81-133.

[8] M. Bromly and C. Hinz. Non-fickian transport in homogeneous unsaturated repacked sand. Water Resour. Res., Vol. 40 ( 2004), W07402, 2004. 
[9] J. Camacho, Thermodynamics of Taylor Dispersion: Constitutive equations, Phys. Rev. E, Vol. 47 (1993), no. 2, pp. 1049-1053.

[10] J. Camacho, Purely Global Model for Taylor Dispersion, Phys. Rev. E, Vol. 48 (1993), no. 1, pp. 310 -321.

[11] J. Camacho, Thermodynamics functions for Taylor's dispersion, Phys. Rev. E, Vol. 48 (1993), no. 3, pp. 1844-1849.

[12] R. Camassa and Z. Lin and R.M. McLaughlin, The exact evolution of the scalar variance in pipe and channel flow, Comm. Math. Sci., Vol. 8 (2010), no. 2, pp. 602-626.

[13] P.C. Chatwin, The initial develoption of longitudial dispersion in straight tubes, J. Fluid Mech., Vol. 80 (1977), pp. 33-48.

[14] C. Choquet and A. Mikelić, Laplace transform approach to the rigorous upscaling of the infinite adsorption rate reactive flow under dominant Peclet number through a pore, Applicable Analysis, Vol. 87 (2008), no. 12., pp. 1373-1395.

[15] C. Choquet and A. Mikelić. Rigorous upscaling of the reactive flow with finite kinetics and under dominant Peclet number, Continuum Mechanics and Thermodynamics, Vol. 21 (2009), pp. 125-140.

[16] A.E. DeGance and L.E. Johns, The theory of dispersion of chemically active solutes in a rectilinear flow field: the vector problem, Appl. Sci. Res., Vol. 42 (1985), pp. 55-88.

[17] C.J. van Duijn, P. Knabner, Travelling Waves in the Transport of Reactive Solutes through Porous Media: Adsorption and Binary Ion Exchange - Part 1, Transport in Porous Media, Vol. 8 (1992), pp. 167-194.

[18] C.J. van Duijn, A. Mikelić, I.S. Pop and C. Rosier, Effective Dispersion Equations For Reactive Flows With Dominant Peclet and Damkohler Numbers, In: Guy B. Marin, David West and Gregory S. Yablonsky, editors: Advances in Chemical Engineering, Vol 34, Academic Press, 2008, pp. $1-45$.

[19] P.C. Fife and K.R.K. Nicholes, Dispersion in flow through small tubes, Proc. Roy. Soc. A, Vol. 344 (1975), pp. 131-145.

[20] W.N. Gill, A note on the solution of transient dispersion problems, Proc. R. Soc. London Ser. A, Vol. 298 (1967), pp. 335-339. 
[21] W.N. Gill and V. Ananthakrishnan, Laminar dispersion in capillaries: Part IV. The slug stimulus, AIChE Journal, Vol. 13 (1967), pp. 801-807.

[22] W.N. Gill and R. Sankarasubramanian, Exact analysis of unsteady convective diffusion, Proc. R. Soc. London Ser. A, Vol. 316 (1970), pp. 341-350.

[23] G. Iosilevskii and H. Brenner, Taylor dispersion in systems containing a continuous distribution of reactive species, Int. J. Nonlinear Mech., Vol. 28 (1993), no. 1, pp. 69-86.

[24] P. Knabner, C.J. van Duijn and S. Hengst, An analysis of crystal dissolution fronts in flows through porous media. Part 1: Compatible boundary conditions, Advances in Water Resources, Vol. 18 (1995), pp. 171185.

[25] M. Latini and A.J. Bernoff, Transient anomalous diffusion in Poiseuille flow, J. Fluid Mech., Vol. 441 (2001), pp. 339-411.

[26] M.J. Lighthill, Initial Development of Diffusion in Poiseuille Flow, IMA J. Appl. Math., Vol. 2 (1966), no.1, pp. 97-108.

[27] G.N. Mercer and A.J. Roberts, A centre manifold description of contaminant dispersion in channels with varying flow profiles, SIAM J. Appl. Math., Vol. 50 (1990), pp. 1547-1565.

[28] R. Metzler and J. Klafter, The restaurant at the end of the random walk, J. Phys. A: Math. Gen., Vol. 37 (2004), pp. 161-208.

[29] A. Mikelić, V. Devigne and C.J. van Duijn, Rigorous upscaling of the reactive flow through a pore, under dominant Peclet and Damkohler numbers, SIAM J. Math. Anal., Vol. 38 (2006), no.4, pp. 1262-1287.

[30] A. Mikelić and C. Rosier, Rigorous upscaling of the infinite adsorption rate reactive flow under dominant Peclet number through a pore, Ann. Univ. Ferrara Sez. VII Sci. Mat., Vol. 53 (2007), no. 2, pp. 333-359.

[31] K.K. Mondal and B.S. Mazumder, On the solute dispersion in a pipe of annular cross-section with absorption boundary, Z. Angew. Math. Mech., Vol. 85 (2005), no. 6, pp. 422-430.

[32] C.O. Ng, Dispersion in steady and oscillatory flows through a tube with reversible and irreversible wall reactions, Proc. R. Soc. A, Vol. 462 (2006), pp. 481-515. 
[33] M.A. Paine, R.G. Carbonell and S. Whitaker, Dispersion in pulsed systems - I, Heterogeneous reaction and reversible adsorption in capillary tubes, Chemical Engineering Science, Vol. 38 (1983), pp. 1781-1793.

[34] O. Pironneau, F. Hecht, A. Le Hyaric and J. Morice, FreeFem ++ version 3.11-0, http://www.freefem.org/ff++/.

[35] M. Quintard and S. Whitaker, Transport in ordered and disordered porous media: volume-averaged equations, closure problems, and comparison with experiment, Chem. Engng. Sci., Vol. 48 (1995), no. 14, pp. 2537-2564.

[36] J. Rubinstein and R. Mauri, Dispersion and convection in porous media, SIAM J. Appl. Math., Vol. 46 (1986), pp. 1018 - 1023.

[37] R. Sankarasubramanian and W.N. Gill, Unsteady convective diffusion with interphase mass transfer, Proc. Roy. Soc. Lond. A, Vol. 333 (1971), pp. 115-132.

[38] A. Sarkar and G. Jayaraman, The effect of wall absorption on dispersion in annular flows, Acta Mech., Vol. 158 (2002), pp. 105-119.

[39] R. Smith, The early stages of contaminant dispersion in shear flows, J. Fluid Mech., Vol. 111 (1981), pp. 107-122.

[40] G.I. Taylor, Dispersion of soluble matter in solvent flowing slowly through a tube, Proc. Royal Soc. A, Vol. 219 (1953), pp. 186-203.

[41] J.S. Vrentas and C.M. Vrentas, Asymptotic solutions for laminar dispersion in circular tubes, Chem. Eng. Sci., Vol. 55 (1967), pp. 849-855. 